\title{
Non-enzymatic lipid oxidation products in biological systems: Assessment of the metabolites from polyunsaturated fatty acids
}

\author{
Claire Vigor ${ }^{\mathrm{a}}$, Justine Bertrand-Michel ${ }^{\mathrm{b}}$, Edith Pinot ${ }^{\mathrm{a}}$, Camille Oger ${ }^{\mathrm{a}}$, Joseph Vercauteren ${ }^{\mathrm{a}}$, \\ Pauline Le Faouder ${ }^{\mathrm{b}}$, Jean-Marie Galano ${ }^{\mathrm{a}}$, Jetty Chung-Yung Lee ${ }^{\mathrm{c}, * *}$, Thierry Durand ${ }^{\mathrm{a}, *}$ \\ a Institut des Biomolécules Max Mousseron IBMM, UMR 5247 CNRS/Université Montpellier 1/Université Montpellier 2, France \\ b Plateau de lipidomique, Bio-Medical Federative Research Institute of Toulouse, INSERM, Plateforme MetaToul, Toulouse, France \\ c The University of Hong Kong, School of Biological Sciences, Hong Kong SAR, China
}

\section{A R T I C L E I N F O}

\section{Article history:}

Received 29 November 2013

Accepted 18 April 2014

Available online 29 April 2014

Keywords:

Oxidative stress

Lipid peroxidation

Isoprostanes

Fatty acid

GC-MS

LC-MS/MS

\begin{abstract}
A B S T R A C T
Metabolites of non-enzymatic lipid peroxidation of polyunsaturated fatty acids notably omega- 3 and omega- 6 fatty acids have become important biomarkers of lipid products. Especially the arachidonic acid-derived $\mathrm{F}_{2}$-isoprostanes are the classic in vivo biomarker for oxidative stress in biological systems. In recent years other isoprostanes from eicosapentaenoic, docosahexaenoic, adrenic and $\alpha$-linolenic acids have been evaluated, namely $\mathrm{F}_{3}$-isoprostanes, $\mathrm{F}_{4}$-neuroprostanes, $\mathrm{F}_{2}$-dihomo-isoprostanes and $\mathrm{F}_{1}$ phytoprostanes, respectively. These have been gaining interest as complementary specific biomarkers in human diseases. Refined extraction methods, robust analysis and elucidation of chemical structures have improved the sensitivity of detection in biological tissues and fluids. Previously the main reliable instrumentation for measurement was gas chromatography-mass spectrometry (GC-MS), but now the use of liquid chromatography-tandem mass spectrometry (LC-MS/MS) and immunological techniques is gaining much attention. In this review, the types of prostanoids generated from non-enzymatic lipid peroxidation of some important omega- 3 and omega- 6 fatty acids and biological samples that have been determined by GC-MS and LC-MS/MS are discussed.
\end{abstract}

Abbreviations: AA, arachidonic acid; AD, Alzheimer disease; AdA, adrenic acid; ALA, $\alpha$-linolenic acid; AMPP, $N$-(4-aminomethylphenyl)pyridinium; APCI, atmospheric pres-

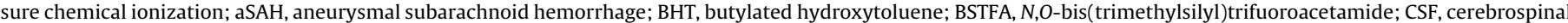

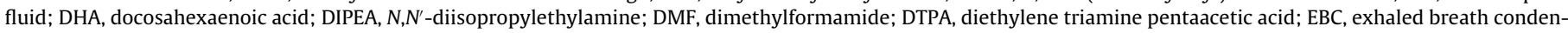

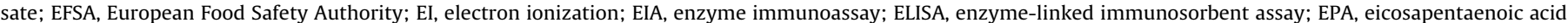

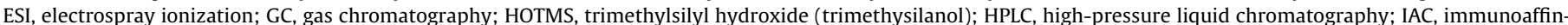

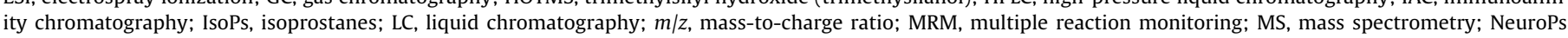

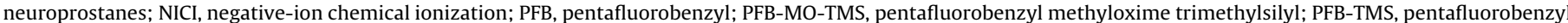

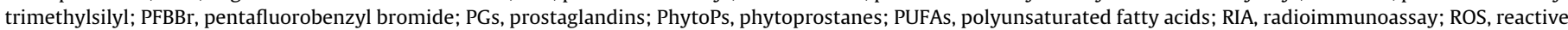

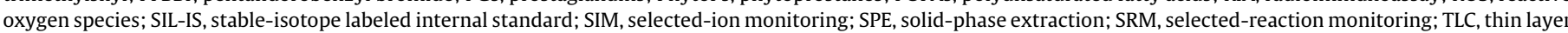
chromatography; TMCS, trimethylchlorosilane; TMS, trimethylsilyl; TPP, triphenylphosphine; UPLC, ultra high-pressure liquid chromatography; UV, ultraviolet.

is This paper is part of the special issues ACIDS edited by Alexander A. Zoerner and Dimitrios Tsikas.

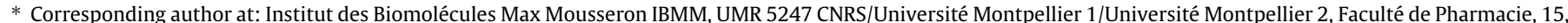
Av. Ch. Flahault, F-34093 Montpellier cedex 05, France. Tel.: +33 4117595 58; fax: +33 411759553.

** Corresponding author at: School of Biological Sciences, The University of Hong Kong, Pokfulam Road, Hong Kong SAR, China. Tel.: +852 22990318.

E-mail addresses: jettylee@hku.hk (J.C.-Y. Lee), Thierry.Durand@univ-montp1.fr (T. Durand). 


\section{Introduction}

Free radicals have been implicated in a number of human diseases such as neurodegenerative, cardiovascular and pulmonary disorders and cancer [1]. Most common free radicals and nonradical species, known as reactive oxygen species (ROS), are able to modify oxidatively lipids, proteins and nucleic acids. Of the lipids in particular the polyunsaturated fatty acids (PUFAs) form a wide variety of oxidized products $[2,3]$.

Among the oxidized lipid products generated, the measurement of isoprostanes (IsoPs) appears to be a promising assay for over two decades due to their specificity and sensitivity for in vivo assessment of oxidative stress and lipid peroxidation [4]. The majority of IsoPs are produced in vivo by non-enzymatic free-radical-induced peroxidation of PUFAs [5]. These compounds are formed in situ on membrane phospholipids and then released in their free form into circulation [6,7]. Depending on the parent PUFAs, different families of IsoPs have been discovered and quantified in several pathological conditions [2]. Among them, $\mathrm{F}_{2}$-IsoPs are the most represented and extensively studied such that to be the designated "gold" marker by laboratories [8], and to be recently validated by the European Food Safety Authority (EFSA) as biomarkers for oxidative damage in cardiovascular health [9].

Various nomenclature guidelines have been proposed in the literature $[10,11]$. In this review, the nomenclature reported by Taber and Roberts [10], validated by the International Union of Pure and Applied Chemistry (IUPAC), will be adopted to describe different non-enzymatic oxidized lipid products of arachidonic, adrenic, eicosapentaenoic, docosahexaenoic, and $\alpha$-linolenic acids. The state-of-the-art analysis of these products, namely $\mathrm{F}_{2}$-IsoPs, $\mathrm{F}_{3}$-isoprostanes ( $\mathrm{F}_{3}$-IsoPs), $\mathrm{F}_{4}$-neuroprostanes ( $\mathrm{F}_{4}$-NeuroPs), $\mathrm{F}_{2}$ dihomo-isoprostanes ( $F_{2}$-dihomo-IsoPs) and $F_{1}$-phytoprostanes (PhytoPs), in biological samples using GC-MS, GC-MS/MS, LC-MS and LC-MS/MS is reviewed (Fig. 1).

\section{Generation of lipid oxidation products}

\subsection{Metabolites of arachidonic acid}

The biosynthesis of the majority of the IsoPs involves a free radical-induced process of arachidonic acid (AA) peroxidation initiated by the presence of radical species mainly centered on the oxygen such as the hydroxyl radical. Generation of prostaglandins (PGs) from free AA is cyclooxygenase dependent, whereas IsoPs made in phospholipid membranes are cyclooxygenase independent. IsoPs are released in their free forms by the platelet-activating factor acetylhydrolase and possibly by other phospholipases $[12,13]$. It was recently found that they circulate predominantly in high-density lipoproteins (HDL) [14] and then subsequently are metabolized and excreted in urine. It was reported that a significant proportion of $\mathrm{F}_{2}$-IsoPs in urine are conjugated as glucuronides [15]. Each series of $\mathrm{F}_{2}$-IsoPs contains eight possible isomers. As each regioisomer includes its racemic counterpart, a total of 64 different $\mathrm{F}_{2}$-IsoPs can be generated; however, it is unknown and not investigated whether these numerous $\mathrm{F}_{2}$-IsoPs generated would have similar properties. Among the $\mathrm{F}_{2}$-IsoPs regioisomers, most human studies have focused on the $15-\mathrm{F}_{2}$-IsoP series, in particular $15-\mathrm{F}_{2 \mathrm{t}}$-IsoP, which is also known as 8 -iso- $\mathrm{PGF}_{2 \alpha}$ and often used as an index for $F_{2}$-IsoPs. Nevertheless, Li and coworkers [16] discovered that $5-\mathrm{F}_{2 \mathrm{t}}$-IsoPs are present in urine at higher concentration than $15-\mathrm{F}_{2 \mathrm{t}}$-IsoPs.

The fundamental difference in the biosynthesis between PGs and IsoPs displays the specificity in the stereostructure of the metabolites. The non-specific initial radical hydrogen atom abstraction at one of the three possible bisallylic positions (7,10 and 13) in AA is the first difference between the IsoPs synthesis and the PGs synthesis, where $\mathrm{H}$-abstraction is regiospecific and occurs only at the 13 position. The subsequent pentadienyl radicals formed in the IsoPs synthesis react with molecular oxygen generating six different pentadienyl peroxyl radicals. Only four of them can produce the four different series of IsoPs compounds via a subsequent irreversible radical cascade (double 5-exo trig cyclization), followed by a final oxygenation leading to $\mathrm{G}_{2}$-IsoPs. Consecutive hydroperoxide and endoperoxide reductions generate the specific $\mathrm{F}_{2}$-IsoPs. The endoperoxide reduction can also be perturbed and following a Kornblum-DeLaMare rearrangement can give E- and DIsoPs. Dehydration of membrane-bound $E_{2}$ - and $D_{2}$-IsoPs is facile under physiological conditions [17] and produces cyclopentenone $\mathrm{A}_{2}$ - and $\mathrm{J}_{2}$-IsoPs, respectively [18]. The particular cis-orientation of the side chains in IsoPs in contrast to trans-orientation in PGs reflects another crucial specificity, linked to the localization of the radical process. For IsoPs biosynthesis, the membrane is the place of action, and therefore conventional chemistry rules apply (lower transition state energy during the double 5-exo-trig cyclization) to generate the 1,2-cis-orientations of the side chains compared to enzymatically driven three-dimensional trans-orientation of the side chains obtained with PGs synthesis. Furthermore, two stereochemistries - the all-syn (represented as subscript " $c$ "; see $5-\mathrm{F}_{2 \mathrm{c}}$-IsoP) and syn-anti-syn stereochemistry (represented as subscript "t"; see $15-\mathrm{F}_{2 \mathrm{t}}$-IsoP) - are present in IsoPs synthesis, and they are well explained by the Beckwing-Houk model of the lower transition states possible during a radical cyclization process; chair- and boat-like transition states are shown in Scheme 1.

The number of theoretical regioisomers further complicates the complexity of IsoPs biosynthesis; the four $\mathrm{F}_{2}$-IsoPs regioisomers having each 8 diastereoisomers could generate 64 racemic compounds. It was shown that the 5- and 15-series IsoPs are the most abundant in vivo, likely due to the fact that the 8- and 12-series IsoPs are more readily oxidized further [19-22].

\subsection{Metabolites of adrenic acid}

Adrenic acid (AdA) is the elongated form of arachidonic acid (AA). Being a two-carbon analog of AA, it will also provide four series of dihomo-isoprostanes (dihomo-IsoPs), the 7- and 17-series being the major metabolites [23]. Similarly to IsoPs, 64 racemic $\mathrm{F}_{2}$ dihomo-IsoPs can theoretically be found in vivo, and so far only the F-series was investigated in biological and analytical studies [23-25].

\subsection{Metabolites of eicosapentaenoic acid}

Eicosapentaenoic acid (EPA) was also found to provide 6 series of $\mathrm{F}_{3}$-IsoPs, the 5 - and 18 -isomers being the most abundant in vivo [26-28]. Theoretically, 96 racemic isomers of $F_{3}$-IsoPs can be observed and quantified as a total in vivo. $\mathrm{F}_{3}$-IsoPs are not the most studied isoprostanoids, probably because EPA is less abundant than AA in human tissues. However, Rokach and coworkers [27,29] have shown that $5-\mathrm{F}_{3}$-IsoP can be quantified in urine and may represent a $\beta$-oxidized metabolite of $7-\mathrm{F}_{4}-\mathrm{NeuroP}$ from DHA $[27,29,30]$.

\subsection{Metabolites of docosahexaenoic acid}

The peroxidation of docosahexaenoic acid (DHA) follows a similar process as described for IsoPs. A total of 8 possible regioisomers identified as 4-, 7-, 10-, 11-, 13-, 14-, 17- and 20-series NeuroPs, and a total of 128 theoretical compounds could be generated. Among these 4- and 20-series represent the two most abundantly found NeuroP isomers in vivo [31]. 

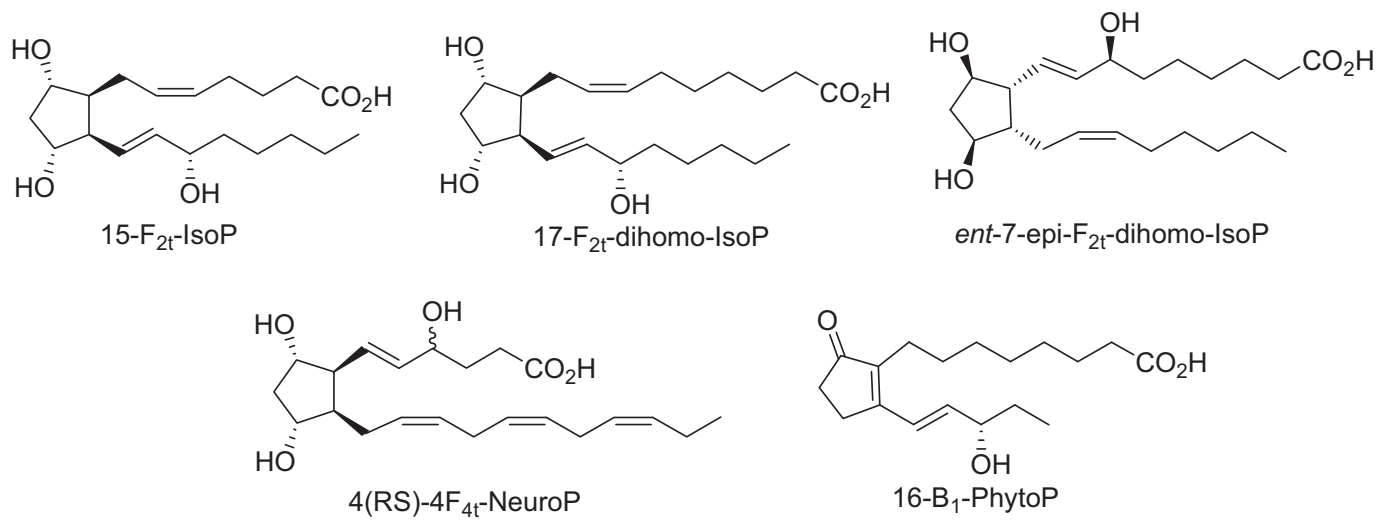

Fig. 1. Representative IsoP, dihomo-IsoP, NeuroP and PhytoP in biological systems.

\subsection{Metabolites of $\alpha$-linolenic acid}

Generation of phytoprostanes (PhytoPs) also involves a free radical-induced reaction of $\alpha$-linolenic acid (ALA) initiated by the active ROS. These PhytoPs are found in plants in which only two series, i.e., the 9- and 16- isomers, are generated [32]. They have also been encountered in human studies after flaxseed oil supplementation [33] and in vegetable oils [34].

\section{Assessment of lipid oxidation products in biological samples}

\subsection{Sample collection}

IsoPs, NeuroPs, PhytoPs and other oxygenated metabolites of n-3 and n-6 PUFAs have been measured in various biological samples. Lipid oxidation products in urine and plasma are indicative of oxidative stress status in vivo and tissue samples. In particular fluids such as exhaled breath condensate (EBC) they represent localized, organ-specific oxidative stress status. For accurate analysis, it is essential to minimize any post-drawing oxidation or degradation after sampling that would affect the measurement. PUFAs, particularly n-3 and n-6 and derivatives, are prone to autoxidation or photodegradation. Therefore, numerous cautions must be taken for sample storage as well as in extraction and derivatization steps. To protect samples from direct sunlight, the use of amber glassware or glassware with aluminum foil is recommended. Samples, especially plasma, cerebrospinal fluid (CSF) and tissues, are commonly excluded from oxygen by flushing with nitrogen or argon, after being treated with suitable antioxidant, such as butylated hydroxytoluene (BHT), triphenylphosphine (TPP), diethylene triamine pentaacetic acid (DTPA) and glutathione (GSH), to inhibit free radicals present in matrix that could generate lipid hydroperoxides artificially and consequently oxidized lipid products.

It is well documented that ultra-low temperatures are necessary for the storage of all biological samples [5] with a restriction

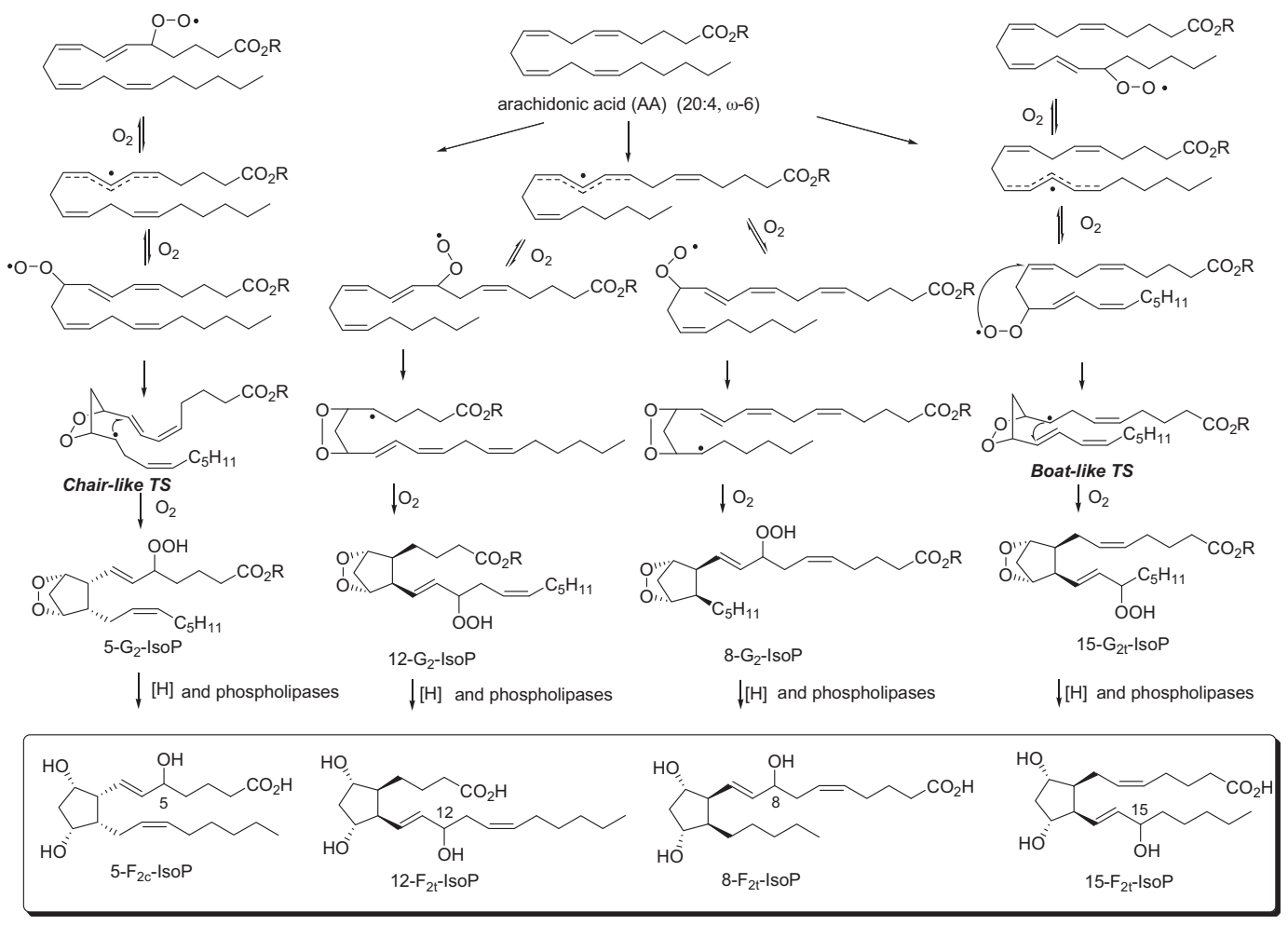

Scheme 1. Biosynthesis of isoprostanes. 

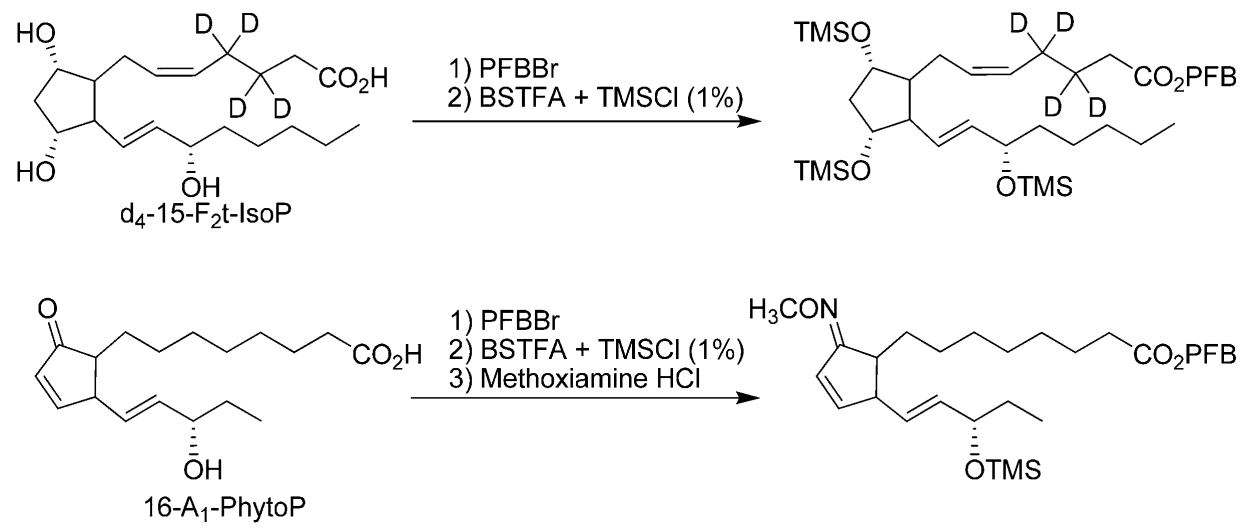

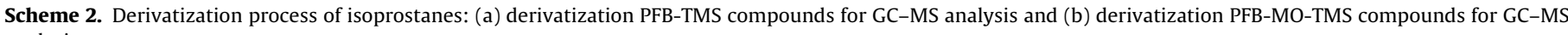
analysis.

of number of freeze-thaw cycles. Recently, it was reported that blood samples should be collected in EDTA/BHT/GSH tubes [35] and it is crucial the plasma portion be stored at $-80^{\circ} \mathrm{C}$ to reduce ex vivo auto-oxidation of $\mathrm{AA}$ that leads to artifact elevation of plasma $\mathrm{F}_{2}$-IsoPs concentrations [5,35]. Urine does not require special processing since it contains only trace quantities of lipids; thus it is not affected by ex vivo formation of n-3 and n-6 PUFA metabolites. However it is suggested to treat it with glucuronidase prior to analysis to release free $\mathrm{F}_{2}$-IsoPs which are accessible to analysis [36]. In general, plasma and tissue samples can be conserved for up to 6 months at $-80^{\circ} \mathrm{C}$ but urine samples can be stored for several years at this temperature [37].

\subsection{Extraction}

Determination of metabolites from lipid peroxidation in biological samples requires one or several preparation steps to extract the compounds from their biological matrix before further analysis by GC-MS, GC-MS/MS, LC-MS, LC-MS/MS or by immunological methods. Sample volumes of biological fluids (plasma, urine, CSF) between 0.2 and $1 \mathrm{~mL}$ [38] are required, while tissues samples between 50 and $100 \mathrm{mg}$ [8,39] and 1-5 million cells are needed [40-42]. IsoPs, NeuroPs or PhytoPs are found to be present in conjugated and free forms endogenously. They can be quantified in their free circulating forms or in total, i.e., the sum of free and esterified, in plasma [43] and tissues after extraction. For tissues or cells, lipids are extracted by homogenizing the samples using Folch solution composed of chloroform and methanol $(2: 1, \mathrm{v} / \mathrm{v})$ with $0.005 \%$ BHT, and then continuously agitated at $4{ }^{\circ} \mathrm{C}$ for 1 hour. Afterwards, $\mathrm{NaCl}(0.9 \%)$ or phosphated buffered saline ( $\mathrm{pH} 7.4)$ is added and the organic lipid layer is extracted for analysis $[4,44]$. For total IsoPs, NeuroPs or PhytoPs measurement, lipid extract is further treated with equal volume of $1 \mathrm{M} \mathrm{KOH}$ prepared in methanol and heated at $37^{\circ} \mathrm{C}$. This step is not required for measurement of 'free' IsoPs, NeuroPs or PhytoPs in plasma, lipid extract from tissues, CSF and urine. As for urine, some authors suggest that pretreatment with glucuronidase can reduce matrix effect and provide cleaner sample for analysis [36].

A purification process is needed to avoid matrix effect and to increase sensitivity of the quantification by LC-MS/MS analysis. Thin layer chromatography (TLC) or solid-phase extraction (SPE) using $\mathrm{C} 18$ [36] or $\mathrm{NH}_{2}$ [45] or anionic exchange phase cartridges $[46,38]$ is commonly used in the purification step. Some methods use a combination of hydrophobic (C18) and pure silica, which has high polarity [4] or $\mathrm{NH}_{2}$ cartridge [45] for the purification. Few authors also combined SPE and TLC in the purification step $[4,47]$. One method proposes purification with a bimodal SPE non-polar cartridge and strong anion exchange to make it specific for analysis of compound of interest [33]. Others suggested the use of HPLC with extraction column [48] or immunoaffinity chromatography (IAC) using immobilized antibodies directed against specific analytes [49]. However, there is a limitation to IAC as it is exclusively commercialized for $15-\mathrm{F}_{2 \mathrm{t}}$-IsoP analysis and not for other metabolites of n-3 and n-6 PUFAs.

\subsection{GC-MS and GC-MS/MS analysis}

Usually, GC involves a sample being vaporized and injected onto the head of the chromatographic column through a hot injector. The sample is transported through the column by the flow of an inert, gaseous mobile phase such as helium. To increase volatility and thermal stability of the analytes, derivatization steps are often required in the sample preparation process. Over the last three decades, considerable improvements have been achieved in extraction and ionization techniques, in particular the development of GC-MS/MS analysis. The use of SPE for purification and isolation considerably improved the signal-to-noise ratio of the GC-MS and highly increased selectivity for compounds.

\subsubsection{Derivatization}

Derivatization of oxidized lipid products is generally performed according to Morrow and Roberts [6]. Briefly, after extraction and preliminary purification steps, the carboxylic acid function of compounds is converted into a pentafluorobenzyl (PFB) ester by reacting samples with pentafluorobenzyl bromide (PFBBr) and $N, N^{\prime}$-diisopropylethylamine (DIPEA) acting as the base catalysts. Free hydroxyl groups are further converted into trimethylsilyl (TMS) ether derivatives by incubating with $\mathrm{N}, \mathrm{O}$ bis(trimethylsilyl)trifluoroacetamide (BSTFA), trimethylchlorosilane (TMCS; 1\%), and dimethylformamide (DMF) leading to the wanted PFB-TMS derivative of the analyte ready for GC-MS analysis. In the specific cases of E-, D-, A- or J-type ring series, carbonyl function is first transformed into $O$-methyloxime derivatives with methoxyamine hydrochloride in pyridine, followed by standard derivatization as shown in Scheme 2 to lead to PFB-MO-TMS compounds.

\subsubsection{Ionization and fragmentation}

Some assays have been developed to improve GC-MS analysis of oxidized lipid products derived from n-3 and n-6 PUFAs with different ionization modes [50]. However, GC-MS in negative-ion chemical ionization (NICI) mode along with selected-ion monitoring (SIM) of a single ion has been shown to be the most reliable analytical approach to assess these compounds for in vivo samples. 
Table 1

Oxidized lipid metabolites and internal standards measured by GC-MS and GC-MS/MS in some studies.

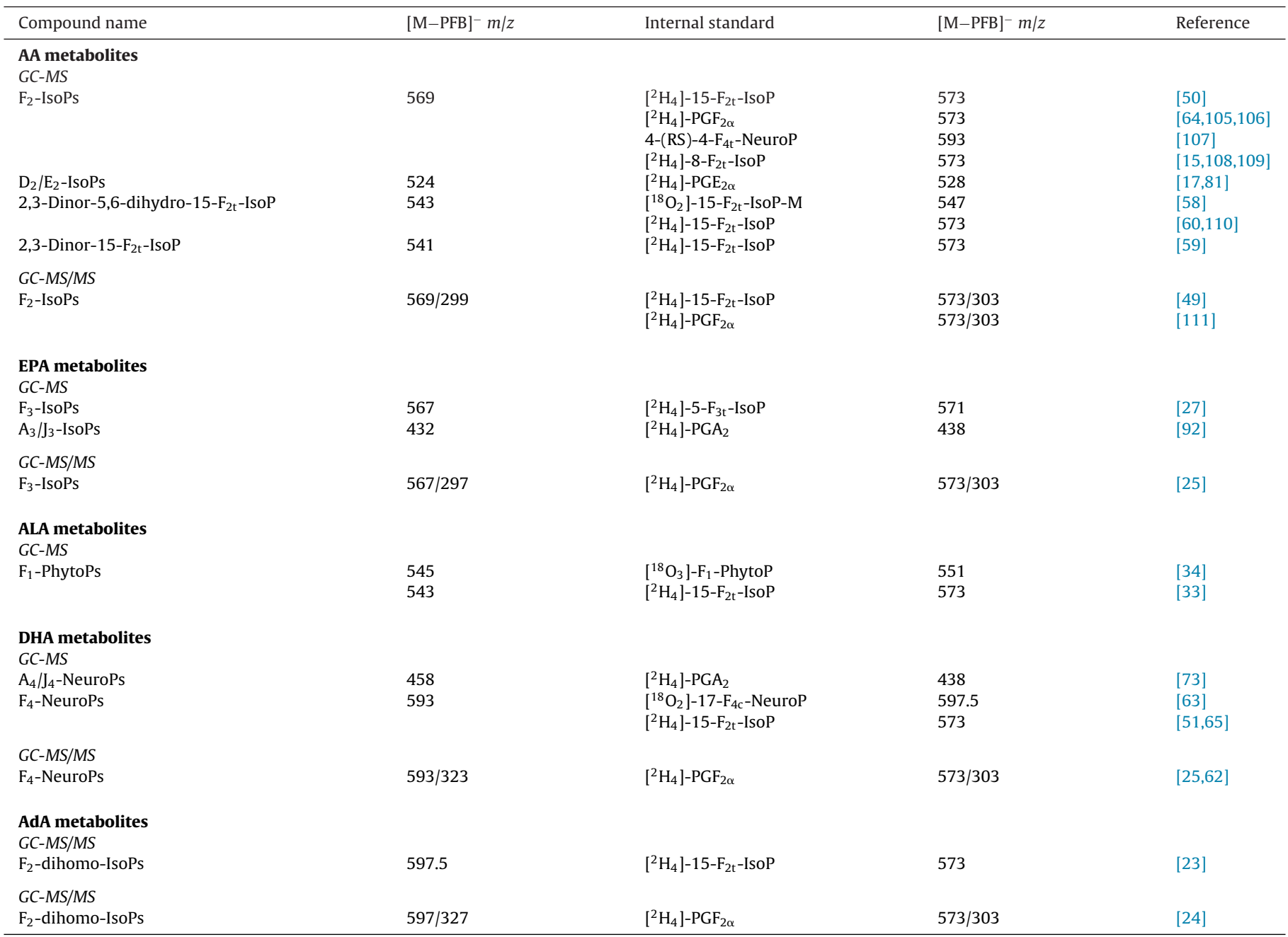

The PFB-TMS or PFB-MO-TMS derivatives of several series of IsoPs, NeuroPs and PhytoPs utilize usually methane as reactant gas and produce mass spectra poor in fragmentations. The spectra are dominated by an intense single ion, the carboxylate anion [M-PFB] ${ }^{-}$ which is formed by cleavage of a highly stabilized PFB radical that follows the capture of an electron by the strong electron capturing PFB moiety of PFB ester. Characteristic [M-PFB $]^{-}$fragments of oxygenated metabolites derived from $n-3$ and n-6 PUFAs such as $\mathrm{F}_{2}$-IsoPs, $\mathrm{F}_{2}$-dihomo-IsoPs, $\mathrm{F}_{3}$-IsoPs, $\mathrm{F}_{4}$-NeuroPs and $\mathrm{F}_{1}$-PhytoPs in human fluids and tissues are summarized in Table 1.

\subsubsection{Metabolites assessed in biological samples}

3.3.3.1. $F_{2}$-isoprostanes. Several GC-MS methods have been applied to a variety of biological sources including urine, plasma, tissue (brain, liver, kidney, colon), CSF, sputum, skin, erythrocytes and fungi $[4,39,51-53]$ (Tables $2 \mathrm{a}$ and 2b). GC-NICI-MS analysis of $\mathrm{F}_{2}$-IsoPs was first developed by Morrow et al. [5] in human plasma and urine, and rat plasma and kidney. In this method, $\mathrm{F}_{2}$-IsoPs are determined by SIM of $m / z 569$ for endogenous $F_{2}$-IsoPs and $\mathrm{m} / \mathrm{z} 573$ for the internal standard $\mathrm{d}_{4}-\mathrm{F}_{2}$-IsoP. The GC-MS method by Morrow et al. [5] is one of the most widely used methods for $\mathrm{F}_{2}$-IsoPs to measure lipid peroxidation and oxidative stress in various clinical studies, mainly vascular related diseases such as atherosclerosis and stroke, and neurodegenerative diseases such as Parkinson and Alzheimer diseases [51,54,55]. In addition, the use of GC-MS for the measurement of $F_{2}$-IsoPs and other
$E_{2} / D_{2}$-IsoPs has also helped to elucidate the biological activities of these products in humans [50].

In 1996, Roberts et al. [56] showed that the major urinary metabolite of $15-\mathrm{F}_{2 \mathrm{t}}$-IsoP in human is 2,3-dinor-5,6-dihydro-15$\mathrm{F}_{2 \mathrm{t}}$-IsoP. Subsequently, Chiabrando et al. [57] found one more metabolite in human urine, 2,3-dinor- $15-\mathrm{F}_{2 \mathrm{t}}-\mathrm{IsoP}$. The sensitive and accurate GC-NICI-MS method developed for IsoPs further allowed the identification and quantitation of these compounds in urine, which are present in higher amounts than the parent $F_{2}$-IsoPs $[57,58]$. A rapid approach to quantify simultaneously urinary $15-\mathrm{F}_{2 \mathrm{t}}$-IsoP and its metabolites $2,3-$ dinor- $15-\mathrm{F}_{2 \mathrm{t}}$-IsoP and 2,3-dinor-5,6-dihydro-15- $\mathrm{F}_{2 \mathrm{t}}$-IsoP prior to final quantitation by GC-NICI-MS was also shown by Nourooz-Zadeh et al. [59]. Measurement of free $\mathrm{F}_{2}$-IsoPs in urine can be confounded by the potential contribution of local $\mathrm{F}_{2}$-IsoPs production in the kidney [51]. Therefore, urinary measurement of the systemic oxidation status in mammals could be best represented by the analysis of these $\mathrm{F}_{2}$-IsoP metabolites as demonstrated by Dorjgochoo et al. [60]. Their results further suggest that 2,3-dinor-5,6-dihydro-15$\mathrm{F}_{2 \mathrm{t}}$-IsoP may be a more sensitive marker of endogenous oxidative stress status than $\mathrm{F}_{2}$-IsoPs in the assessment of antioxidants' effects on age-related diseases.

Measurements of $\mathrm{F}_{2}$-IsoPs by GC-NICI-MS are not limited to biological fluids such as plasma and urine. It has been determined in sputum to investigate oxidative stress and pulmonary disorders, in amniotic fluids in pre-eclampsia pregnancy and in CSF in 
Table 2a

Metabolites measured in biological fluids by GC-MS and GC-MS/MS in some studies.

\begin{tabular}{|c|c|c|c|c|c|c|c|c|}
\hline Sample & Study & Organism & $\begin{array}{l}\text { Sample } \\
\text { preparation }\end{array}$ & $\begin{array}{l}\text { Precursor fatty } \\
\text { acid }\end{array}$ & $\begin{array}{l}\text { Precursor/product } \\
\text { ion } m / z\end{array}$ & Metabolite & Baseline level $^{\mathrm{a}}$ & Reference \\
\hline $\begin{array}{l}\text { Plasma } \\
\text { Urine }\end{array}$ & Healthy & Human & SPE: MAX & AA & 569 & $\mathrm{~F}_{2}$-IsoPs & $\begin{array}{l}239 \pm 21 \mathrm{pg} / \mathrm{mL} \\
502 \pm 404 \mathrm{pg} / \mathrm{mg} \mathrm{Cr}\end{array}$ & [38] \\
\hline $\begin{array}{l}\text { Plasma } \\
\text { Urine }\end{array}$ & Healthy & Human & LLE and SPE: Si & ALA & 545 & $\begin{array}{l}\mathrm{F}_{1} \text {-PhytoPs } \\
\text { Plasma } \\
\text { Urine }\end{array}$ & $\begin{array}{l}<\text { LOD } \\
<\text { LOD }\end{array}$ & [112] \\
\hline $\begin{array}{l}\text { Plasma } \\
\text { Urine }\end{array}$ & $\begin{array}{l}\text { Flaxseed oil } \\
\text { supplemen- } \\
\text { tation to } \\
\text { healthy } \\
\text { subjects }\end{array}$ & Human & SPE: Certify II & $\begin{array}{l}\text { AA } \\
\text { ALA }\end{array}$ & $\begin{array}{l}569 \\
543\end{array}$ & $\begin{array}{l}\mathrm{F}_{2} \text {-IsoPs } \\
\text { Plasma } \\
\text { Urine } \\
\mathrm{F}_{1} \text {-PhytoPs } \\
\text { Plasma } \\
\text { Urine }\end{array}$ & $\begin{array}{l}1.99 \pm 0.17 \mathrm{nmol} / \mathrm{L} \\
10.30 \pm 1.54 \mathrm{nmol} / 24 \mathrm{~h} \\
4 \mathrm{nmol} / \mathrm{L}^{\mathrm{b}} \\
275 \mathrm{nmol} / 24 \mathrm{~h}^{\mathrm{b}}\end{array}$ & [33] \\
\hline $\begin{array}{l}\text { Plasma } \\
\text { Urine }\end{array}$ & $\begin{array}{l}\text { Overweight } \\
\text { Dislipidemia } \\
\text { Type } 2 \text { diabetes }\end{array}$ & Human & SPE: Certify II & AA & 569 & $\begin{array}{l}\mathrm{F}_{2} \text {-IsoPs } \\
\text { Plasma } \\
\text { Urine }\end{array}$ & $\begin{array}{l}207.1 \pm 7.8 \mathrm{pg} / \mathrm{mL} \\
2100 \mathrm{pg} / \mathrm{mg} \mathrm{Cr}\end{array}$ & {$[108,109]$} \\
\hline $\begin{array}{l}\text { Plasma } \\
\text { Urine }\end{array}$ & $\begin{array}{l}\text { Age-matched } \\
\text { control and } \\
\text { Parkinson's disease } \\
\text { (stage 1) }\end{array}$ & Human & SPE: MAX & DHA & 593 & $\begin{array}{l}\mathrm{F}_{2} \text {-IsoPs } \\
\text { Control } \\
\text { Plasma } \\
\text { Urine } \\
\mathrm{PD} \\
\text { Plasma } \\
\text { Urine } \\
\mathrm{F}_{4} \text {-NeuroPs } \\
\text { Control } \\
\text { Plasma } \\
\text { PD } \\
\text { Plasma }\end{array}$ & $\begin{array}{l}0.36 \pm 12 \mathrm{ng} / \mathrm{mL} \\
0.54 \pm 0.27 \mathrm{ng} / \mathrm{mg} \mathrm{Cr} \\
0.76 \pm 0.30 \mathrm{ng} / \mathrm{mL} \\
0.57 \pm 0.26 \mathrm{ng} / \mathrm{mg} \mathrm{Cr} \\
0.45 \pm 0.25 \mathrm{ng} / \mathrm{mL} \\
0.67 \pm 0.24 \mathrm{ng} / \mathrm{mL}\end{array}$ & [113] \\
\hline $\begin{array}{l}\text { Plasma } \\
\text { Urine }\end{array}$ & Healthy & Human & $\begin{array}{l}\text { SPE: C-18, Si } \\
\text { TLC }\end{array}$ & AA & 569 & $\begin{array}{l}\mathrm{F}_{2} \text {-IsoPs } \\
\text { Plasma } \\
\text { Urine }\end{array}$ & $\begin{array}{l}35 \pm 6 \mathrm{pg} / \mathrm{mL} \\
1600 \pm 600 \mathrm{pg} / \mathrm{mg} \mathrm{Cr}\end{array}$ & {$[4]$} \\
\hline Plasma & $\mathrm{CCl}_{4}$ treatment & Rat & $\begin{array}{l}\text { SPE: C-18 } \\
\text { TLC }\end{array}$ & AA & 569 & $\mathrm{~F}_{2}$-IsoPs & $430 \pm 70 \mathrm{pg} / \mathrm{mL}$ & [8] \\
\hline Plasma & $\begin{array}{l}\text { Healthy } \\
\text { Dengue fever } \\
\text { Parkinson's } \\
\text { disease } \\
\text { Ischemic stroke }\end{array}$ & Human & SPE: MAX & AA & 569 & $\begin{array}{l}\mathrm{F}_{2} \text {-IsoPs } \\
\text { Healthy } \\
\text { Dengue } \\
\text { fever } \\
P D \\
\text { Ischemic } \\
\text { stroke }\end{array}$ & $\begin{array}{l}0.58 \pm 0.29 \mathrm{ng} / \mathrm{mL} \\
0.59 \pm 0.22 \mathrm{ng} / \mathrm{mL} \\
0.72 \pm 0.28 \mathrm{ng} / \mathrm{mL} \\
0.94 \pm 0.36 \mathrm{ng} / \mathrm{mL}\end{array}$ & [55] \\
\hline Plasma & Rett syndrome & Human & SPE: $\mathrm{C}-18, \mathrm{NH}_{2}$ & $\begin{array}{l}\text { AA } \\
\text { AdA } \\
\text { EPA } \\
\text { DHA }\end{array}$ & $\begin{array}{l}567 / 297 \\
569 / 299 \\
593 / 323 \\
597 / 327\end{array}$ & $\begin{array}{l}\mathrm{F}_{2} \text {-IsoPs } \\
\mathrm{F}_{2} \text {-dihomo- } \\
\text { IsoPs } \\
\mathrm{F}_{3} \text {-IsoPs } \\
\mathrm{F}_{4} \text {-NeuroPs }\end{array}$ & $\begin{array}{l}144 \mathrm{pg} / \mathrm{mL} \\
1.0 \pm 0.11 \mathrm{pg} / \mathrm{mL} \\
15.0 \pm 10 \mathrm{pg} / \mathrm{ml}^{\mathrm{b}} \\
1.0 \mathrm{pg} / \mathrm{mL}\end{array}$ & $\begin{array}{l}{[24,25,62,} \\
114]\end{array}$ \\
\hline Urine & $\begin{array}{l}\text { Polytraumatized } \\
\text { patients }\end{array}$ & Human & SPE: $\mathrm{C}-18, \mathrm{NH}_{2}$ & AA & 569 & $\mathrm{~F}_{2}$-IsoPs & $811 \pm 359 \mathrm{pg} / \mathrm{mg} \mathrm{Cr}$ & [107] \\
\hline $\begin{array}{l}\text { Plasma } \\
\text { Cord blood }\end{array}$ & $\begin{array}{l}\text { Pregnant women } \\
\text { Pre-eclampsia }\end{array}$ & Human & SPE: Certify II & $\begin{array}{l}\text { AA } \\
\text { DHA }\end{array}$ & $\begin{array}{l}569 \\
593\end{array}$ & $\begin{array}{l}\mathrm{F}_{2} \text {-IsoPs } \\
\mathrm{F}_{4} \text {-NeuroPs }\end{array}$ & $\begin{array}{l}727 \mathrm{pg} / \mathrm{mL} \\
731 \mathrm{pg} / \mathrm{mL}\end{array}$ & [67] \\
\hline Urine & Healthy & Human & SPE: Certify II & AA & 569 & $\mathrm{~F}_{2}$-IsoPs & $1904 \pm 94 \mathrm{pg} / \mathrm{mg} \mathrm{Cr}$ & {$[36]$} \\
\hline Platelets & $\begin{array}{l}\text { Human platelets } \\
\text { aggregation }\end{array}$ & Human & SPE: Certify II & AA & 569 & $\begin{array}{l}\mathrm{F}_{2} \text {-IsoPs } \\
8-\mathrm{F}_{2 \mathrm{t}} \text {-IsoP }\end{array}$ & $\begin{array}{l}50 \mathrm{fmol}^{\mathrm{b}} \\
250 \mathrm{fmol}^{\mathrm{b}}\end{array}$ & [115] \\
\hline CSF & $\begin{array}{l}\text { Aneurismal } \\
\text { subarachnoid } \\
\text { hemorrhage }\end{array}$ & Human & $\begin{array}{l}\text { SPE: C-18, Si } \\
\text { TLC }\end{array}$ & DHA & 593 & $\mathrm{~F}_{4}$-NeuroPs & $77.8 \pm 19.5 \mathrm{pg} / \mathrm{mL}$ & {$[51]$} \\
\hline CSF & $\begin{array}{l}\text { Inspired oxygen during } \\
\text { limb surgery }\end{array}$ & Human & SPE: Certify II & $\begin{array}{l}\text { AA } \\
\text { DHA }\end{array}$ & $\begin{array}{l}585 \\
593\end{array}$ & $\begin{array}{l}\mathrm{F}_{2} \text {-IsoPs } \\
\mathrm{F}_{4} \text {-NeuroPs }\end{array}$ & $\begin{array}{l}400 \mathrm{pmol} / \mathrm{L}^{\mathrm{b}} \\
300 \mathrm{pmol} / \mathrm{L}^{\mathrm{b}}\end{array}$ & [65] \\
\hline Sputum & $\begin{array}{l}\text { Inflammatory airway } \\
\text { diseases }\end{array}$ & Human & $\begin{array}{l}\text { C18 Sep-Pak } \\
\text { Cartridge }\end{array}$ & AA & 569 & $\mathrm{~F}_{2}$-IsoP & Not reported & {$[116]$} \\
\hline
\end{tabular}

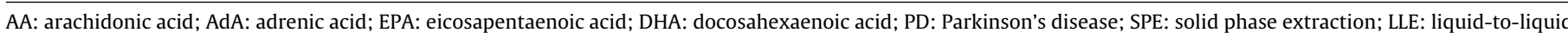
extraction; MAX: mixed anionic exchange; Certify II: mixed mode/strong anionic exchange; TLC: thin layer chromatography; Cr: creatinine.

a Baseline values indicate healthy group, age-matched group, pre-treatment prior intervention or initial stage of disease development.

b Approximate reading from graphical display reported in the literature. 
Table 2b

Metabolites measured in biological cells and tissues by GC-MS and GC-MS/MS in some studies.

\begin{tabular}{|c|c|c|c|c|c|c|c|c|}
\hline Sample & Study & Organism & $\begin{array}{l}\text { Sample } \\
\text { preparation }\end{array}$ & $\begin{array}{l}\text { Precursor fatty } \\
\text { acid }\end{array}$ & $\begin{array}{l}\text { Precursor/product } \\
\text { ion } m / z\end{array}$ & Metabolite & Baseline level $^{\mathrm{a}}$ & Reference \\
\hline $\begin{array}{l}\text { Liver } \\
\text { Kidney }\end{array}$ & $\begin{array}{l}\text { Effects of age and } \\
\text { caloric restriction }\end{array}$ & Rat & $\begin{array}{l}\text { SPE: C-18, Si } \\
\text { TLC }\end{array}$ & $\mathrm{AA}$ & 569 & $\begin{array}{l}\mathrm{F}_{2} \text {-IsoPs } \\
\text { Liver } \\
\text { Kidney }\end{array}$ & $\begin{array}{l}10.34 \pm 0.85 \mathrm{ng} / \mathrm{g} \\
5.04 \pm 0.49 \mathrm{ng} / \mathrm{g}\end{array}$ & [117] \\
\hline $\begin{array}{l}\text { Brain } \\
\text { Liver, } \\
\text { erythrocytes }\end{array}$ & Antioxidants & Mouse & SPE: $\mathrm{C}-18, \mathrm{NH}_{2}$ & $\mathrm{AA}$ & 481 & $\begin{array}{l}\mathrm{F}_{2} \text {-IsoPs } \\
\text { Brain } \\
\text { Liver } \\
\text { Erythrocytes }\end{array}$ & $\begin{array}{l}29.78 \mathrm{ng} / \mathrm{g} \\
22.08 \mathrm{ng} / \mathrm{g} \\
4.68 \mathrm{ng} / \mathrm{mL}\end{array}$ & [39] \\
\hline $\begin{array}{l}\text { Liver } \\
\text { Liver }\end{array}$ & $\begin{array}{l}\text { Hypercholesterolemia } \\
\text { PGs formation via IsoPs } \\
\text { pathway }\end{array}$ & $\begin{array}{l}\text { Rabbit } \\
\text { Rat }\end{array}$ & $\begin{array}{l}\text { SPE: ion exchange } \\
\text { SPE: C-18, Si, HPLC }\end{array}$ & $\begin{array}{l}\text { AA } \\
\text { AA }\end{array}$ & $\begin{array}{l}569 \\
524\end{array}$ & $\begin{array}{l}\mathrm{F}_{2} \text {-IsoPs } \\
\mathrm{PGE}_{2} / \mathrm{D}_{2}\end{array}$ & $\begin{array}{l}1.3 \pm 0.3 \mathrm{ng} / \mathrm{mg} \\
1240 \pm 450 \mathrm{ng} / \mathrm{mg} \\
\text { arachidonate }\end{array}$ & $\begin{array}{l}{[118]} \\
{[119]}\end{array}$ \\
\hline Brain & $\begin{array}{l}\text { Oxidative stress } \\
\text { induction with oxygen }\end{array}$ & Pig & SPE: C-18, Si & $\begin{array}{l}\text { AA } \\
\text { DHA }\end{array}$ & $\begin{array}{l}569 \\
593\end{array}$ & $\begin{array}{l}\mathrm{F}_{2} \text {-IsoPs } \\
\mathrm{F}_{4} \text {-NeuroPs }\end{array}$ & $\begin{array}{l}6.05 \pm 2.0 \mathrm{ng} / \mathrm{g} \\
23.27 \pm 6.6 \mathrm{ng} / \mathrm{g}\end{array}$ & [120] \\
\hline Brain & $\begin{array}{l}\text { Alzheimer's } \\
\text { disease }\end{array}$ & $\begin{array}{l}\text { Mouse } \\
\text { Human }\end{array}$ & $\begin{array}{l}\text { SPE: C-18, Si } \\
\text { TLC }\end{array}$ & DHA & 593 & $\begin{array}{l}\mathrm{F}_{4}-\mathrm{NeuroPs} \\
\text { Mouse brain } \\
\text { Human brain }\end{array}$ & $\begin{array}{l}8.7 \pm 2.0 \mathrm{ng} / \mathrm{g} \\
4.9 \pm 0.6 \mathrm{ng} / \mathrm{g}\end{array}$ & {$[68]$} \\
\hline Brain & $\begin{array}{l}\text { Alzheimer's } \\
\text { disease }\end{array}$ & Human & $\begin{array}{l}\text { SPE } \\
\text { TLC }\end{array}$ & $\mathrm{AA}$ & 569 & $\begin{array}{l}\mathrm{F}_{2} \text {-IsoPs } \\
\text { Frontal } \\
\text { Temporal } \\
\text { Occipital } \\
\text { Cerebellum }\end{array}$ & $\begin{array}{l}159 \pm 36 \mathrm{pg} / \mathrm{mg} \\
154 \pm 27 \mathrm{pg} / \mathrm{mg} \\
110 \pm 15 \mathrm{pg} / \mathrm{mg} \\
118 \pm 20 \mathrm{pg} / \mathrm{mg}\end{array}$ & {$[54]$} \\
\hline Brain & Healthy & Human & $\begin{array}{l}\text { SPE: } \mathrm{C}-18, \mathrm{Si} \\
\text { TLC }\end{array}$ & $\begin{array}{l}\text { AA } \\
\text { DHA } \\
\text { AdA }\end{array}$ & $\begin{array}{l}569 \\
593 \\
597\end{array}$ & $\begin{array}{l}\mathrm{F}_{2} \text {-IsoPs } \\
\mathrm{F}_{4} \text {-NeuroPs } \\
\mathrm{F}_{2} \text {-dihomo- } \\
\text { IsoPs }\end{array}$ & $\begin{array}{l}1.5 \pm 0.1 \mathrm{ng} / \mathrm{g} \\
11.3 \pm 0.6 \mathrm{ng} / \mathrm{g} \\
14.5 \pm 0.5 \mathrm{ng} / \mathrm{g}\end{array}$ & {$[23]$} \\
\hline $\begin{array}{l}\text { Skin } \\
\text { microdialysis }\end{array}$ & Healthy female & Human & SPE: C-18 & $\mathrm{AA}$ & $\begin{array}{l}569 \\
569\end{array}$ & $\begin{array}{l}\mathrm{F}_{2} \text {-IsoPs } \\
9 \alpha-11 \alpha- \\
\mathrm{PGF}_{2 \alpha}\end{array}$ & $\begin{array}{l}0.28 \pm 0.06 \mathrm{pmol} / \mathrm{mL} \\
0.65 \pm 0.08 \mathrm{pmol} / \mathrm{mL}\end{array}$ & [52] \\
\hline $\begin{array}{l}\text { Catfish } \\
\text { epidermal } \\
\text { secretion }\end{array}$ & Healthy & Fish & $\begin{array}{l}\text { LLE } \\
\text { TLC }\end{array}$ & $\mathrm{AA}$ & 569 & $\begin{array}{l}\mathrm{F}_{2} \text {-IsoPs } \\
\text { Male } \\
\text { Female }\end{array}$ & $\begin{array}{l}4.17-5.63 \mathrm{ng} / \mathrm{mL} \\
3.60-3.81 \mathrm{ng} / \mathrm{mL}\end{array}$ & [121] \\
\hline $\begin{array}{l}\text { Aspergillus } \\
\text { fumigatus }\end{array}$ & $\begin{array}{l}\text { Production of } \\
\text { eicosanoids }\end{array}$ & Fungus & IAC & AA & $\begin{array}{l}569 / 299 \\
524 / 268 \\
569 / 299 \\
524 / 268 \\
524 / 268\end{array}$ & $\begin{array}{l}15-\mathrm{F}_{2 \mathrm{t}} \text {-IsoP } \\
15-\mathrm{E}_{2 \mathrm{t}} \text {-IsoP } \\
\mathrm{PGF}_{2 \alpha} \\
\mathrm{PGE}_{2} \\
\mathrm{PGD}_{2}\end{array}$ & $\begin{array}{l}<1-5.6 \mathrm{pg} / \mathrm{mL} \\
<1 \mathrm{pg} / \mathrm{mL} \\
<1 \mathrm{pg} \text { to } 17.7 \mathrm{pg} / \mathrm{mL} \\
<1 \mathrm{pg} / \mathrm{mL} \\
<1 \mathrm{pg} / \mathrm{mL}\end{array}$ & {$[53]$} \\
\hline
\end{tabular}

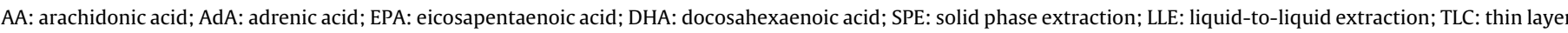
chromatography; IAC: immunoaffinity column.

a Baseline values indicate healthy group, age-matched group, pre-treatment prior intervention or initial stage of disease development.

aneurysmal subarachnoid hemorrhage (Table 2a). Various biological tissues have been analyzed for $\mathrm{F}_{2}$-IsoPs, mainly animal liver and brain are investigated in toxicological studies and neurodegenerative diseases respectively. Both liver and kidney tissues of rats were also analyzed to understand the effect of age and calorie restriction and its relationship to DNA oxidation. Analysis of $\mathrm{F}_{2}$-IsoPs in biological samples in recent years further expanded in human skin after UV light exposure, in fish epidermis and even in fungi (Table 2b).

3.3.3.2. $F_{2}$-dihomo-isoprostanes. In 2008, VanRollins et al. [23] described the formation of F-ring IsoPs from adrenic acid (AdA, $\mathrm{C} 22: 4, \mathrm{n}-6)$. These compounds were named $\mathrm{F}_{2}$-dihomo-IsoPs. AdA, like DHA, is highly enriched in the brain but is primarily found in white matter and is associated with oxidative stress in the myelin portion. From the results provided by GC-MS analysis, it was deduced that these compounds could be considered as indicators of oxidative stress-driven white matter damages in human brain. For example, phospholipids derived from human cerebral gray matter, white matter, and myelin were similarly oxidized ex vivo and showed the ratio of esterified $\mathrm{F}_{2}$-dihomo-IsoPs to $\mathrm{F}_{4}$-NeuroPs approximately 10 -fold greater in myelin-derived than in gray matter-derived phospholipids. The study suggests that quantification of dihomo-IsoPs may be a selective marker of white matter injury in vivo. De Felice et al. [24] systematically investigated the impact of AdA and non-enzymatic peroxidation in plasma of Rett patients (Table 2a). For this study two dihomo-

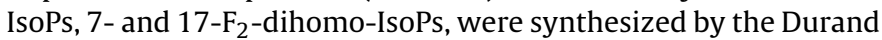
team [61], and accurately measured by GC-NICI-MS(MS) based on their previous method [62] after SPE (C18 and $\mathrm{NH}_{2}$ cartridge) and standard derivatization steps. It was found that average plasma $\mathrm{F}_{2}$-dihomo-IsoP levels in Rett syndrome were approximately two orders of magnitude higher at early stage of the disease followed by a steady decrease during the natural clinical progression [24] (Table 2a).

3.3.3.3. $F_{3}$-isoprostanes. The development of $\mathrm{F}_{3}$-IsoPs in vivo from EPA was identified and reported by Nourooz-Zadeh et al. [26] and Gao et al. [28]. Quantitation of total $F_{3}$-IsoPs level is performed by determining merged peaks of $m / z 567$ signal and using $\mathrm{d}_{4}-\mathrm{F}_{2}$-IsoP as SIL-IS in GC-NICI-MS analysis. Since physiological EPA concentration is very low, endogenous level of $\mathrm{F}_{3}$-IsoPs in tissues of animals and humans is reported to be approximately $<30 \mathrm{pg} / \mathrm{g}$ of tissue only. Apart from the above two reports very little information [25] is available concerining assessing $\mathrm{F}_{3}$-IsoPs levels by using GC-NICIMS.

3.3.3.4. Neuroprostanes. Docosahexaenoic acid (DHA, C22:6, n-3) is concentrated in the brain and enriched in neurons; therefore, 

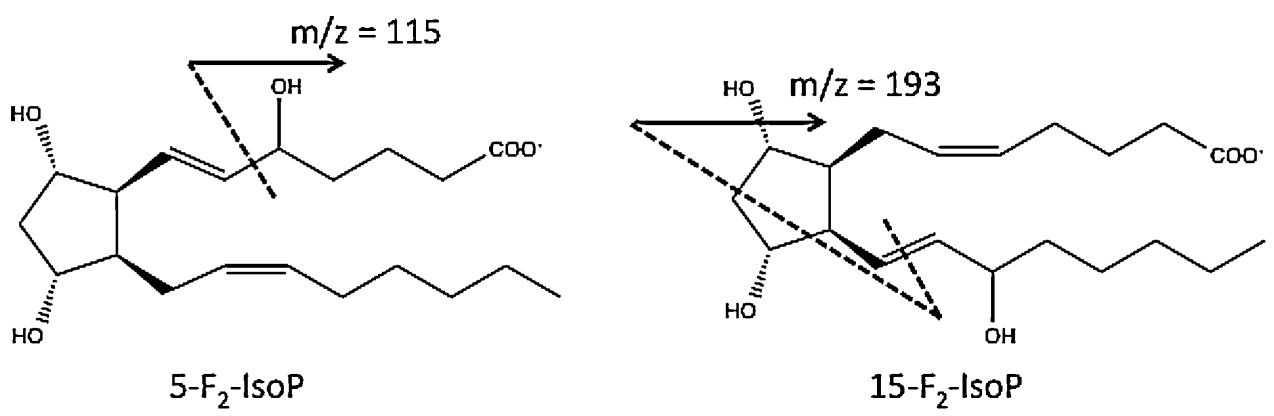

$15-F_{2}-$ IsoP

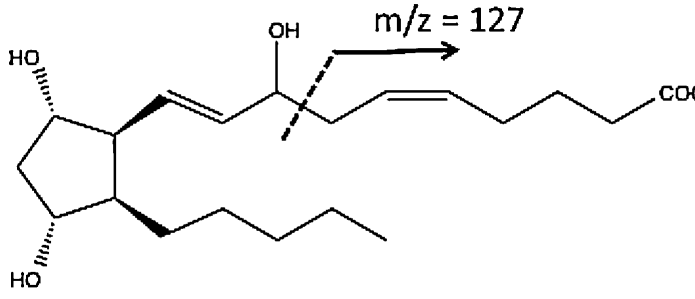

$8-F_{2}-$ IsoP

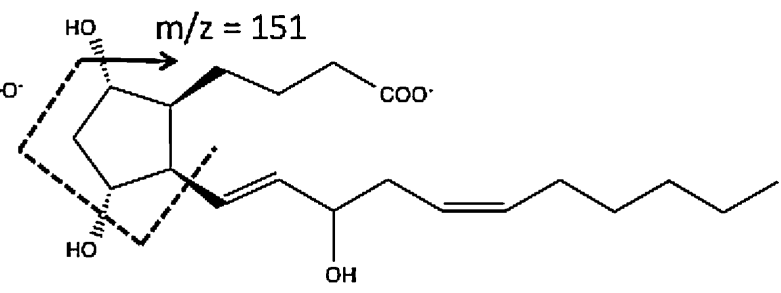

$12-F_{2}-$ IsoP

Fig. 2. Characteristic fragmentation of four series of $\mathrm{F}_{2}$-IsoPs.

$\mathrm{F}_{4}$-NeuroPs are considered as the most selective indicator for oxidative stress and neurodegenerative diseases [55]. In 1998, Roberts et al. [63] showed that the presence of $F_{4}$-NeuroPs has no counterpart in the PG family (in contrary to AA, EPA and AdA). They showed that the metabolite is purely from free radical-mediated lipid peroxidation of DHA, both in vitro and in vivo, and measurable with the same GC-NICI-MS method as $\mathrm{F}_{2}$-IsoPs [63]. $\mathrm{F}_{4}$-NeuroPs is determined by merged peaks of $\mathrm{m} / \mathrm{z} 593$ signal and using $\left[{ }^{18} \mathrm{O}_{2}\right]-17-\mathrm{F}_{4 \mathrm{C}}-\mathrm{NeuroP},\left[{ }^{2} \mathrm{H}_{4}\right]-15-\mathrm{F}_{2 \mathrm{t}}$-IsoP or $\left[{ }^{2} \mathrm{H}_{4}\right]-\mathrm{PGF}_{2} \alpha$ as SIL-IS in GC-NICI-MS analysis.

It was found by Nourooz-Zadeh et al. [64] that the levels of $\mathrm{F}_{4}$-NeuroPs were 4 -fold higher than $\mathrm{F}_{2}$-IsoP levels in plasma of Alzheimer disease (AD) patients. Hsieh et al. [51] and others [65] detected $\mathrm{F}_{2}$-IsoPs and $\mathrm{F}_{4}$-NeuroPs in CSF using $\left[{ }^{2} \mathrm{H}_{4}\right]-15-\mathrm{F}_{2 \mathrm{t}}$-IsoP as IS and analyzed by GC-NICI-MS in aneurysmal subarachnoid hemorrhage $(\mathrm{aSAH})$ patients. Their results suggest that $\mathrm{F}_{4}-\mathrm{NeuroPs}$ in CSF could be a better predictor for outcome of aSAH than $\mathrm{F}_{2}$-IsoPs at early stage. They also indicated that the analysis of $\mathrm{F}_{4}$ NeuroPs that use $\left[{ }^{18} \mathrm{O}_{2}\right]-17-\mathrm{F}_{4 \mathrm{c}}-\mathrm{NeuroP}$ as SIL-IS could potentially interfere with $\mathrm{F}_{2}$-dihomo-IsoPs chromatographic peak in CSF samples $[51,66]$. Nevertheless, the same GC-NICI-MS method was also applied to elucidate the role of $\mathrm{F}_{2}$-IsoPs, IsoFs and $\mathrm{F}_{4}$-NeuroPs in pre-eclampsia and normal pregnancy maternal plasma and cord blood plasma [67] (Table 2a). Again, measurement of $\mathrm{F}_{4}$-NeuroPs is not restricted to biological fluids. Results by Musiek et al. [68] showed an increase of 2.1 -fold of $\mathrm{F}_{4}$-NeuroPs in the temporal lobe of AD patients' brain compared to that in control samples. They developed an assay using synthesized $\left[{ }^{18} \mathrm{O}_{2}\right]-17-\mathrm{F}_{4 \mathrm{c}}-$ NeuroP as SILIS and showed low limit of quantification at approximately $10 \mathrm{pg}$ by GC-NICI-MS analysis.

Signorini et al. [69] also developed a GC-MS/MS method to quantify $\mathrm{F}_{4}$-NeuroPs; extraction, purification and derivatization procedures of $\mathrm{F}_{2}$-IsoPs and $\mathrm{F}_{4}$-NeuroPs from brain tissue were adapted from Nourooz-Zadeh et al. method [64], then compounds were analyzed by GC-NICI-MS(MS) using $\left[{ }^{2} \mathrm{H}_{4}\right]-\mathrm{PGF}_{2 \alpha}$ as SIL-IS. The MS/MS spectrum of the brain sample was dominated by ions produced by loss of trimethylsilyl hydroxide (HOTMS). Evidently, ions at $m / z 503,413$ and 323 were attributable to elimination of one, two or three HOTMS groups, respectively, from the ions [M-PFB $]^{-}$of $\mathrm{F}_{4}{ }^{-}$ NeuroPs. Similar fragmentation and neutral loss of HOTMS were reported for the SIL-IS $\left(m / z\right.$ 303) and $\mathrm{F}_{2}$-IsoPs $(\mathrm{m} / z$ 299). With this GC-NICI-MS(MS) method, the detection limit was approximately $10 \mathrm{pg} / \mathrm{mL}$ for plasma [62].

Reports also showed that $\mathrm{E}_{4}$-NeuroPs and $\mathrm{D}_{4}$-NeuroPs can be detected by GC-NICI-MS in normal rat and human brains [70], and interestingly the F-ring to D/E-ring ratio for NeuroPs, but not IsoPs was $40-70 \%$ lower in all brain regions of $A D$ patients compared to controls [71]. Indeed, the levels of $\mathrm{E}_{4} / \mathrm{D}_{4}$-NeuroPs in normal brain are found to be one-third compared to levels of $\mathrm{F}_{4}$-NeuroPs [72]. $\mathrm{J}_{4} / \mathrm{A}_{4}$-NeuroPs were also analyzed by GC-NICI-MS with $\left[{ }^{2} \mathrm{H}_{4}\right]-\mathrm{PGA}_{2}$ as SIL-IS after SPE and derivatization to PFB-MO-TMS compounds [73]. The major ions present in the NICI mass spectra of $A_{4} / J_{4}$ NeuroPs, and the $\left[{ }^{2} \mathrm{H}_{4}\right]-\mathrm{PGA}_{2}$ derivatives are the carboxylate anions at $m / z 458$ and $m / z 438$, respectively.

3.3.3.5. Phytoprostanes. In 1998, Parchmann and Mueller [74] were the first ones to discover the formation of dinor- $\mathrm{E}_{1}$-IsoP from ALA in plants. These compounds named $E_{1}$-phytoprostanes ( $E_{1}$-PhytoPs) were initially analyzed by GC-EI-MS after derivatization to the PFB ester and TMS ether derivatives. Thereafter, the use of GC-NICIMS and $\left[{ }^{18} \mathrm{O}_{1}\right]-\mathrm{B}_{1}$-PhytoP as SIL-IS enabled the separation of the two regioisomers of $\mathrm{B}_{1}$-PhytoPs chromatographically. Following this discovery, Imbusch and Mueller [32] showed $F_{1}$-PhytoPs could be quantified by separating $F_{1}$-PhytoPs to the acyclic triols (derived from both enzymatic and non-enzymatic oxidation of linolenate in plants) since these compounds had identical chromatographic and mass spectrometric properties with same molecular weight. The only difference between the two types is that $F_{1}$-PhytoPs have one double bond compared to two for acyclic compounds. Thus, during the sample preparation step, and after SPE extraction, the eluate was hydrogenated before derivatization. In this report, the most intense ion identified is the $[\mathrm{M}-\mathrm{PFB}]^{-}$without double bond at $m / z 545$. Nevertheless, $\mathrm{A}_{1}$-PhytoPs and $\mathrm{B}_{1}$-PhytoPs quantification include two main challenges: (1) the A-ring-to-Bring isomerization at higher temperature (GC injector port) and (2) the low derivatization ability in combination with isotope SIL-IS becomes unstable during analysis. Consequently, Thoma et al. [75] proposed this measurement in two steps using GC-NICI-MS. The first analysis conduces to the determination of the $A_{1}$-PhytoPs and $\mathrm{B}_{1}$-PhytoPs sum by using $\left[{ }^{18} \mathrm{O}_{1}\right]-\mathrm{B}_{1}$-PhytoP as SIL-IS. The second 


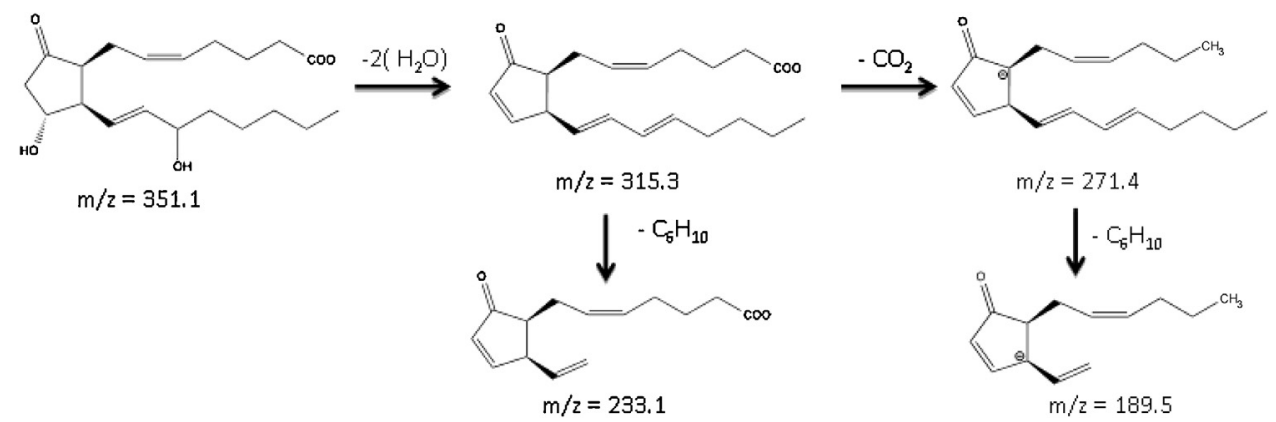

Scheme 3. Characteristic product ions of $E_{2}$-IsoP fragmentations.

analysis, performed after methyloximation of the keto function, is to prevent isomerization of $A_{1}$-PhytoP into $B_{1}$-PhytoP. From the combined information of the two experiments, $A_{1}$-PhytoP and $B_{1}$ PhytoP respective quantitation could be calculated.

PhytoP levels were also measured in human blood and urine before and after plant oil consumption [34]. Interestingly, it was found that total $\mathrm{F}_{1}$-PhytoPs circulating in plasma levels were below the limit of detection of GC-MS analysis but urinary $\mathrm{F}_{1}$-PhytoP was elevated. In 2009, Barden et al. [33] analyzed simultaneously levels of $\mathrm{F}_{2}$-IsoPs and $\mathrm{F}_{1}$-PhytoPs in plasma and urine of male subjects to investigate the effect of flaxseed oil supplementation (Table 2a). They developed a facile extraction/separation method with SPE before derivatization for GC-NICI-MS analysis. The $\mathrm{F}_{1}$-PhytoPs are measured by monitoring $\mathrm{m} / \mathrm{z} 543$ signal and comparing to the SIL-IS $\left[{ }^{18} \mathrm{O}_{1}\right]-\mathrm{F}_{1}-$ PhytoP. The results showed that after four weeks supplementation with flaxseed oil, plasma ALA phospholipids were significantly increased and highly correlated with increased $\mathrm{F}_{1}$ PhytoPs in plasma and urine. Although PhytoPs have been reported to possess a broad spectrum of biological activities in different plant species [76], further studies are required to determine physiological activities in human diseases.

\subsection{LC-MS and LC-MS/MS analysis}

LC is able to separate different kinds of oxygenated metabolites of PUFAs and allows multiple compound evaluation in one analysis. To date, there is no report on the levels of PhytoPs measured by LC. In the following part, we will not distinguish performances between high-pressure liquid Chromatography (HPLC), ultra highpressure liquid chromatography (UHPLC), micro- or nano-flow HPLC, but we will concentrate on their respective use.

Sample preparation procedures, such as extraction, derivatization or purification, are often critical and time-consuming and may lead to a substantial loss of target compounds, or transformation of them by ex vivo autoxidation. Compared to GC, LC presents the advantage to omit derivatization steps and also no compound vaporization for elution is necessary, thus limiting the risk of deterioration for thermosensitive compounds. With LC methodology, sample preparation usually consists of a single extraction step, so analyte recovery may be higher by one order of magnitude [36,37]. However, derivatization of eicosanoids in LC-MS/MS may improve sensitivity as outlined below. Because the sample preparation protocols are relatively generic, it is easy to analyze metabolites across multiple classes resulting in even greater timesaving as shown by Massey [77] or Shinde [78]. However, strong matrix effect and signal suppression can be observed. Unfortunately, these parameters are not systematically studied and reported in the literature. A report by Prasain et al. [46] indicated a total recovery yield of $86 \%$ for urinary eicosanoids, after accounting for extraction, matrix and suppression ion effects. Also, Sterz et al. [79] reported that liquid/liquid extraction procedure could replace SPE in UPLC-MS analysis of urinary eicosanoids.

\subsubsection{Ionization and fragmentation}

For lipid analysis, electrospray ionization (ESI) is widely used and usually operated in negative ionization mode which generates carboxylate ions $[\mathrm{M}-\mathrm{H}]^{-}$from any "free" IsoPs. An alternative to ESI is the atmospheric pressure chemical ionization (APCI) which minimizes matrix effect such as ion suppression [48,80]. From an analytical point of view, also shown by others, the four classes of $\mathrm{F}_{2}$-IsoPs produce the same intermediate ion (carboxylate anion) at $m / z$ 353, under ESI conditions in the negative-ion mode [50]. After collision-induced dissociation of the molecular ion, product ions below $m / z 200$ differ between various isomers: $m / z 115$ for $5-\mathrm{F}_{2 \mathrm{t}}$-IsoP, $m / z 127$ for $8-\mathrm{F}_{2 \mathrm{t}}$-IsoP, $m / z 151$ for $12-\mathrm{F}_{2 \mathrm{t}}$-IsoP and $\mathrm{m} / \mathrm{z} 193$ for $15-\mathrm{F}_{2 \mathrm{t}}$-IsoP [50] (Fig. 2). These intermediate transitions allow selected-reaction monitoring (SRM) of $m / z 353$ (parent ion) to $\mathrm{m} / \mathrm{z} 193$ (product ion). A characteristic SRM for 15-series $\mathrm{F}_{2 \mathrm{t}}$-IsoPs is shown in Fig. 2. Thus, SRM can be employed to differentiate between the four regioisomers. Although studies of IsoPs formation have mainly focused on $\mathrm{F}_{2}$-IsoPs, other IsoPs such as Eand D-IsoPs have been investigated as well [81]. However, only few groups have developed LC-MS or LC-MS/MS methods for the study of these metabolites which share two main specific multiple reaction monitoring (MRM) transitions $m / z 351 \rightarrow 271$ and $m / z$ $351 \rightarrow 189$, but also the additional MRM transitions $m / z 351 \rightarrow 233$, $m / z 351 \rightarrow 315$, and $m / z 351 \rightarrow 333$ (Scheme 3) [37,82,83]. One limitation of LC-ESI-MS(MS) is the missing sensitivity which is in the $10-20 \mathrm{pg}$ range. Charge reversal derivatization was tested [84-86] by which the carboxylic acid is converted to an amide bearing a permanent positive charge [84-86]. Recent improvement by Bollinger et al. [87] highlighted the use of commercially available AMPP ( $N$-(4-aminomethylphenyl)pyridinium). In combination with LC-ESI-MS(MS) in positive ion mode, this derivatization method improved the sensitivity for eicosanoids by one order of magnitude compared to standard protocols.

\subsubsection{Analytical application in biological samples}

3.4.2.1. $F_{2}$-isoprostanes. In the firstly reported LC-MS/MS study on the separation and identification of $\mathrm{F}_{2}$-IsoP regioisomers and diastereoisomers, Yin et al. [88] showed that the 15-series and the 5-series of IsoPs diastereoisomers with cis-alkyl chains on the prostane ring are formed in greater amount than those with transconfiguration. Precisely, these diastereoisomers are also formed to a greater extent than the 8 - and 12-compounds probably because only the transient peroxyl radical intermediates of the 8- and 12series of $\mathrm{F}_{2}$-IsoPs can form a novel class of IsoPs termed dioxolane IsoPs [21]. For the past decade, interest in $F_{2}$-IsoPs measurement by LC-MS/MS and more recently by UHPLC-MS/MS has grown, especially in $15-\mathrm{F}_{2 \mathrm{t}}$-IsoP determinations. While it is not the most abundant isoprostanes in human urine [89], it is the most 
Table 3a

Metabolites measured in biological fluids by LC-MS and LC-MS/MS in some studies.

\begin{tabular}{|c|c|c|c|c|c|c|c|c|}
\hline Sample & Study & Organism & Sample preparation & $\begin{array}{l}\text { Precursor fatty } \\
\text { acid }\end{array}$ & {$[\mathrm{M}-\mathrm{H}]^{-} m / z$} & Metabolite & Baseline level $^{\mathrm{a}}$ & Reference \\
\hline $\begin{array}{l}\text { Plasma } \\
\text { Urine } \\
\text { Full blood }\end{array}$ & Healthy & Human & SPE: C-18 & $\mathrm{AA}$ & 353 & $\begin{array}{l}15(\mathrm{RS})-15-\mathrm{F}_{2 \mathrm{t}}-\mathrm{IsoP} \\
\text { Plasma } \\
\text { Urine } \\
\text { Full blood }\end{array}$ & $\begin{array}{l}40-170 \mathrm{pg} / \mathrm{mL} \\
30-303 \mathrm{ng} / \mathrm{g} \mathrm{Cr} \\
1-2 \mathrm{ng} / \mathrm{mL}\end{array}$ & [122] \\
\hline Plasma & $\begin{array}{l}\text { Normotensive } \\
\text { pregnant } \\
\text { women }\end{array}$ & Human & LLE & $\mathrm{AA}$ & $\begin{array}{l}353 \\
353 \\
353 \\
353 \\
353 \\
353 \\
353\end{array}$ & 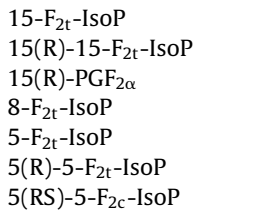 & $\begin{array}{l}195 \mathrm{pg} / \mathrm{mL} \\
177 \mathrm{pg} / \mathrm{mL} \\
338 \mathrm{pg} / \mathrm{mL} \\
137 \mathrm{pg} / \mathrm{mL} \\
416 \mathrm{pg} / \mathrm{mL} \\
393 \mathrm{pg} / \mathrm{mL} \\
879 \mathrm{pg} / \mathrm{mL}\end{array}$ & [123] \\
\hline Plasma & $\begin{array}{l}\text { Aspirin } \\
\text { administration in } \\
\text { male healthy } \\
\text { subjects }\end{array}$ & Human & SPE: C-8 & $\mathrm{AA}$ & 353 & $\begin{array}{l}15-\mathrm{F}_{2 \mathrm{t}}-\mathrm{IsoP} \\
\text { (32 arachidonic acid } \\
\text { related metabolites) }\end{array}$ & $52 \pm 26 \mathrm{pg} / \mathrm{mL}$ & [78] \\
\hline Urine & Smokers & Human & $\begin{array}{l}\text { SPE: } \\
\text { C-18/hydro }\end{array}$ & $\mathrm{AA}$ & $\begin{array}{l}353 \\
325 \\
327\end{array}$ & $\begin{array}{l}\text { 15- } \mathrm{F}_{2 \mathrm{t}} \text {-IsoP } \\
\text { 2,3-Dinor-15-F } \mathrm{F}_{2 \mathrm{t}} \text {-IsoP } \\
\text { 2,3-Dinor-5,6-dihydro- } \\
\text { 15- } \mathrm{F}_{2 \mathrm{t}} \text {-IsoP }\end{array}$ & $\begin{array}{l}200-1500 \mathrm{pg} / \mathrm{mg} \\
\mathrm{Cr} \\
3000-5000 \mathrm{pg} / \mathrm{mg} \\
\mathrm{Cr} \\
1000-3000 \mathrm{pg} / \mathrm{mg} \\
\mathrm{Cr}\end{array}$ & {$[124]$} \\
\hline Urine & Cardiac surgery & Human & $\begin{array}{l}\text { SPE: polymeric } \\
\text { weak anion } \\
\text { exchange }\end{array}$ & AA & $\begin{array}{l}353 \\
353\end{array}$ & $\begin{array}{l}15-\mathrm{F}_{2 \mathrm{t}}-\mathrm{IsoP} \\
15(\mathrm{R})-15-\mathrm{F}_{2 \mathrm{t}} \text {-IsoP }\end{array}$ & $\begin{array}{l}395 \pm 51 \mathrm{pg} / \mathrm{mg} \\
\mathrm{Cr} \\
477 \pm 81 \mathrm{ng} / \mathrm{mg} \\
\mathrm{Cr}\end{array}$ & {$[46]$} \\
\hline Urine & Smokers & Human & $\begin{array}{l}\text { LLE } \\
\text { SPE: } \\
\text { C-18/polymeric/weak/ } \\
\text { strong anion exchange }\end{array}$ & $\mathrm{AA}$ & $\begin{array}{l}353 \\
325\end{array}$ & $\begin{array}{l}\text { 15- } \mathrm{F}_{2 \mathrm{t}}-\mathrm{IsoP} \\
\text { 2,3-Dinor-15- } \mathrm{F}_{2 \mathrm{t}}-\mathrm{IsoP}\end{array}$ & $\begin{array}{l}178 \mathrm{pg} / \mathrm{mg} \mathrm{Cr} \\
1080 \mathrm{pg} / \mathrm{mg} \mathrm{Cr}\end{array}$ & [79] \\
\hline CSF & $\begin{array}{l}\text { Neuro-degenerative } \\
\text { disorders }\end{array}$ & Human & SPE: C-18 & $\mathrm{AA}$ & $\begin{array}{l}353 \\
353\end{array}$ & $\begin{array}{l}15-\mathrm{F}_{2 \mathrm{t}}-\mathrm{IsoP} \\
5-\mathrm{F}_{2 \mathrm{t}}-\mathrm{IsoP}\end{array}$ & $\begin{array}{l}5-80 \mathrm{pg} / \mathrm{mL} \\
12.5-250 \mathrm{pg} / \mathrm{mL}\end{array}$ & [91] \\
\hline $\begin{array}{l}\text { Exhaled } \\
\text { breath } \\
\text { condensate }\end{array}$ & Asbestosis & Human & SPE: IAC & $\mathrm{AA}$ & 353 & $15-\mathrm{F}_{2 \mathrm{t}}$-IsoP & $36 \mathrm{pg} / \mathrm{mL}$ & {$[125]$} \\
\hline $\begin{array}{l}\text { Exhaled breath } \\
\text { condensate }\end{array}$ & Smokers & Human & No preparation & $\mathrm{AA}$ & 353 & $\begin{array}{l}15-F_{2 t}-\text { IsoP } \\
15(R)-15-F_{2 t}-I s o P \\
\text { PGs }\end{array}$ & $\begin{array}{l}<\text { LOD } \\
<\text { LOD } \\
<\text { LOD }\end{array}$ & {$[126]$} \\
\hline $\begin{array}{l}\text { Amniotic } \\
\text { fluid }\end{array}$ & Preeclampsia & Human & HPLC C-18 partition & $\mathrm{AA}$ & 353 & $15-\mathrm{F}_{2 \mathrm{t}}$-IsoP & $73.8 \pm 36.6 \mathrm{pg} / \mathrm{mL}$ & [97] \\
\hline
\end{tabular}

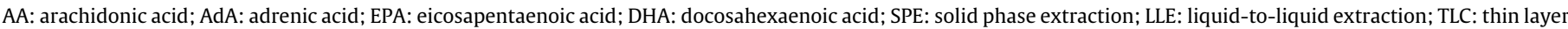
chromatography; IAC: immunoaffinity column; $\mathrm{Cr}$ : creatinine.

a Baseline values indicate healthy group, age-matched group, pre-treatment prior intervention or initial stage of disease development.

thoroughly investigated $\mathrm{F}_{2}$-IsoPs [90] and it is assessed in other biological fluids such as urine, plasma, CSF and EBC (Table 3a). Analogous to GC-MS, tissue samples obtained from the brain, liver, kidney, Caenorhabditis elegans, fish muscle and carcinoma cells have been analyzed by LC-MS/MS in various studies (Table 3b). Human brain and liver samples were also analyzed by LC-MS/MS in different studies [91].

3.4.2.2. $F_{3}$-isoprostanes. Only a few papers describe the LC-MS/MS measurement of $\mathrm{F}_{3}$-IsoPs formed from the oxidation of EPA in vivo. In 2006, Lawson et al. [29] proposed LC-MS/MS quantification of $5-\mathrm{F}_{3 \mathrm{t}}$-IsoP in human urine, and they discovered the $\beta$-oxidation of $7-\mathrm{F}_{4 \mathrm{t}}-\mathrm{NeuroP}$ to $5-\mathrm{F}_{3 \mathrm{t}}$-IsoP. The same year, other groups have identified $\mathrm{F}_{3}$-IsoPs in heart tissues of EPA-supplemented mice [28] and showed that the 5 - and 18 -series $\mathrm{F}_{3}$-IsoPs are the most abundant type in vivo. More recently the same group has demonstrated the generation of $\mathrm{A}_{3}$ - and $\mathrm{J}_{3}$-IsoPs from the oxidation of EPA using an almost identical approach [92]. Furthermore, the Rokach group $[27,30]$ showed that the all-syn stereoisomer, 5- $\mathrm{F}_{3 \mathrm{c}}$-IsoP and its 5-epimer are the major $\mathrm{F}_{3}$-metabolites in human urine. In other biological samples, Chung et al. [93] quantified $\mathrm{F}_{3}$-IsoP in toxicological study of marine fish, and Leung et al. [42] assessed it in sonoporated Jurkat cells. A recent study using UHPLC-MS/MS methodology for quantification of endogenous oxidative damage in the nematode $C$. elegans found $\mathrm{F}_{3}$-IsoPs as the best biomarker for lipid peroxidation by ROS $[40,45]$ (Table $3 b)$.

3.4.2.3. $F_{4}$-neuroprostanes. Initially, LC-MS/MS studies on $\mathrm{F}_{4}$ NeuroPs mainly focused on structural information, specifically the regiochemistry of $\mathrm{F}_{4}$-NeuroPs formation after autoxidation of DHA [31]. In addition to $\mathrm{F}_{4}$-NeuroPs, the highly unstable $\mathrm{E}_{4^{-}}$and $\mathrm{D}_{4^{-}}$ NeuroPs which lead to the highly reactive $\mathrm{A}_{4}$ - and $\mathrm{J}_{4}$-NeuroPs were also investigated using a variety of complementary chemical (GSH adducts formation) and MS approaches using LC-ESI-MS(MS) but mainly for identification rather than quantification $[71,73]$. Neuroprostanes have been measured in mice and rat brains in studies related to neurodegenerative disorders [82,83]. Chung et al. [93] showed $\mathrm{F}_{4}$-NeuroPs can be quantified by LC-MS/MS in marine fish muscle and in Jurkat cells by Leung et al. [42] (Table 3b). 
Table 3b

Metabolites measured in biological cells and tissues by LC-MS and LC-MS/MS in some studies.

\begin{tabular}{|c|c|c|c|c|c|c|c|c|}
\hline Sample & Study & Organism & $\begin{array}{l}\text { Sample } \\
\text { preparation }\end{array}$ & $\begin{array}{l}\text { Precursor } \\
\text { fatty acid }\end{array}$ & {$[\mathrm{M}-\mathrm{H}]^{-} m / z$} & Metabolite & Baseline level $^{\mathrm{a}}$ & Reference \\
\hline $\begin{array}{l}\text { Kidney } \\
\text { Liver }\end{array}$ & $\mathrm{CCl}_{4}$ treatment & Rat & LLE & AA & 353 & $\begin{array}{l}15-\mathrm{F}_{2 \mathrm{t}}-\mathrm{IsoP} \\
\text { Kidney } \\
\text { Liver } \\
\text { 5- } \mathrm{F}_{2 \mathrm{c}} \text {-IsoP } \\
\text { Kidney } \\
\text { Liver }\end{array}$ & $\begin{array}{l}6.5 \mathrm{ng} / \mathrm{g} \\
8.4 \mathrm{ng} / \mathrm{g} \\
20.6 \mathrm{ng} / \mathrm{g} \\
43.9 \mathrm{ng} / \mathrm{g}\end{array}$ & [127] \\
\hline Liver & $\begin{array}{l}\text { Hepatitis C or B virus } \\
\text { infected patient }\end{array}$ & Human & LLE & AA & 353 & $15-\mathrm{F}_{2 \mathrm{t}}-\mathrm{IsoP}$ & $24.78 \mathrm{ng} / \mathrm{g}$ & [13] \\
\hline Brain & Ischemia & Rat & SPE: HLB & AA & 351 & $\mathrm{E}_{2} / \mathrm{D}_{2}$-IsoPs & $1300 \pm 317 \mathrm{pg} / \mathrm{g}$ & [82] \\
\hline Brain & $\begin{array}{l}\text { Neuro-degenerative } \\
\text { disorders }\end{array}$ & Human & SPE: C-18 & AA & $\begin{array}{l}353 \\
353\end{array}$ & $\begin{array}{l}15-\mathrm{F}_{2 \mathrm{t}}-\mathrm{IsoP} \\
5-\mathrm{F}_{2 \mathrm{t}} \text {-IsoP }\end{array}$ & $\begin{array}{l}30-70 \mathrm{pg} / 0.1 \mathrm{~g} \\
60-150 \mathrm{pg} / 0.1 \mathrm{~g}\end{array}$ & [91] \\
\hline Brain & Ischemia & Mouse & LLE & AA & 351 & $\begin{array}{l}15-E_{2 t}-\text { IsoP } \\
E_{2} / D_{2}-P G s\end{array}$ & $1400 \mathrm{pg} / \mathrm{gww}$ & [83] \\
\hline Whole animal & Wild or mutant type & $\begin{array}{l}\text { Nematode } \\
\text { C. elegans }\end{array}$ & $\begin{array}{l}\text { LLE } \\
\text { SPE: } \mathrm{NH}_{2}\end{array}$ & EPA & 351 & $\mathrm{~F}_{3}$-IsoPs & $10 \mathrm{pg} / \mu \mathrm{g}$ protein ${ }^{\mathrm{b}}$ & {$[40]$} \\
\hline Muscle tissue & $\begin{array}{l}\text { Hydrogen } \\
\text { peroxide } \\
\text { treatment }\end{array}$ & Medaka fish & SPE: MAX & $\begin{array}{l}\text { EPA } \\
\text { DHA }\end{array}$ & $\begin{array}{l}353 \\
325 \\
327\end{array}$ & 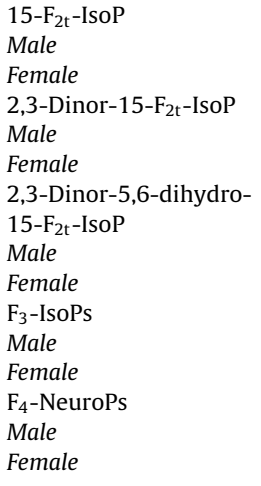 & $\begin{array}{l}1.88 \pm 0.11 \mathrm{ng} / \mathrm{g} \\
3.18 \pm 0.21 \mathrm{ng} / \mathrm{g} \\
2.40 \pm 0.37 \mathrm{ng} / \mathrm{g} \\
9.70 \pm 0.19 \mathrm{ng} / \mathrm{g} \\
\\
119.80 \pm 9.47 \mathrm{ng} / \mathrm{g} \\
50.0 \pm 6.22 \mathrm{ng} / \mathrm{g} \\
0.86 \pm 0.07 \mathrm{ng} / \mathrm{g} \\
2.46 \pm 0.26 \mathrm{ng} / \mathrm{g} \\
0.84 \pm 0.18 \mathrm{ng} / \mathrm{g} \\
0.55 \pm 0.06 \mathrm{ng} / \mathrm{g}\end{array}$ & [93] \\
\hline $\begin{array}{l}\text { Carcinoma } \\
\text { cell line }\end{array}$ & $\begin{array}{l}\text { Pro- or antioxidant } \\
\text { treatment }\end{array}$ & Human & $\begin{array}{l}\text { LLE } \\
\text { SPE: } \mathrm{NH}_{2}\end{array}$ & $\mathrm{AA}$ & $\begin{array}{l}353 \\
353\end{array}$ & $\begin{array}{l}15-\mathrm{F}_{2 \mathrm{t}}-\mathrm{IsoP} \\
5-\mathrm{F}_{2 \mathrm{t}} \text {-IsoP }\end{array}$ & $\begin{array}{l}8 \mathrm{pg}^{\mathrm{b}} \\
50 \mathrm{pg}^{\mathrm{b}}\end{array}$ & [128] \\
\hline Jurkat cell line & Sonoporation & Human & SPE: MAX & $\begin{array}{l}\text { AA } \\
\text { EPA } \\
\text { DHA }\end{array}$ & $\begin{array}{l}353 \\
351 \\
377\end{array}$ & $\begin{array}{l}15-\mathrm{F}_{2 \mathrm{t}}-\mathrm{IsoP} \\
\mathrm{F}_{3}-\mathrm{IsoPs} \\
\mathrm{F}_{4} \text {-NeuroPs }\end{array}$ & $\begin{array}{l}2.26 \mathrm{ng} / \mathrm{ng} \text { arachidonate } \\
0.35 \mathrm{ng} / \mathrm{ng} \\
\text { eicosa-pentaenoate } \\
0.29 \mathrm{ng} / \mathrm{ng} \\
\text { docosa-hexaenoate }\end{array}$ & {$[42]$} \\
\hline $\begin{array}{l}\text { Lung epithelial } \\
\text { cell line }\end{array}$ & Nanoparticle & Human & SPE: HLB & AA & $\begin{array}{l}353 \\
353\end{array}$ & $\begin{array}{l}15-F_{2 t}-\text { IsoP } \\
15(R)-15-F_{2 t}-\text { IsoP }\end{array}$ & $\begin{array}{l}0.0294 \pm 0.0007 \mathrm{ng} / \text { million } \\
\text { cells } \\
0.036 \pm 0.0023 \mathrm{ng} / \text { million } \\
\text { cells }\end{array}$ & [41] \\
\hline Erythrocytes & Healthy & Human & SPE: C-18 & AA & 353 & $15(\mathrm{RS})-15-\mathrm{F}_{2 \mathrm{t}}$-IsoP & $0.4-1.1 \mathrm{ng} / \mathrm{mL}$ & [122] \\
\hline
\end{tabular}

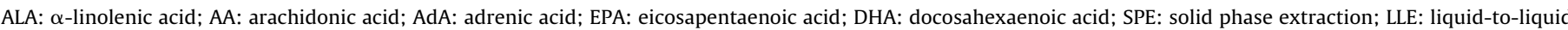
extraction; MAX: mixed anionic exchange; HLB: hydrophilic-lipophilic-balanced; TLC: thin layer chromatography; Cr: creatinine.

a Baseline values indicate healthy group, age-matched group, pre-treatment prior intervention or initial stage of disease development.

b Estimated reading from graphical display reported in the literature.

\subsection{Immunological methods}

Enzyme immunoassay (EIA) and radioimmunoassay (RIA) techniques are based on the same principle, i.e., on the immunological reaction between an antibody that recognizes and binds a specific antigen (analyte) in the sample being tested. The underlying basic principle of immunoassay is competitive binding, where a "tracer" competes with the "analyte" for a fixed number of antibodies. Eicosanoid concentrations are calculated by means of calibration curves which are prepared with the respective synthetic eicosanoid. The type of detection, i.e., the choice of the tracer, makes the only difference between EIA and RIA with the use of an antigen linked to an enzyme able to induce a colorimetric reaction or the use of a radioactive antigen, respectively.

Theoretically, because each antibody is prepared against one specific antigen, only certain metabolites can be measured at a time. In practice, however, because of the large number of different oxidation products formed in vivo from lipids, and because of their very slight discrepancies in their structures and properties, cross-reactivity is common. Cross-reactivity can vary from "negligible" (less than $1 \%$ ) to $10 \%$ or even exceptionally high to $20 \%$ for some eicosanoids. This issue was investigated in detail by Basu [94] and Wang et al. [95], who determined cross-reactivity for a number of synthetic $\mathrm{F}_{2}$-IsoPs using EIA and RIA. Klawitter et al. [80] compared the quantification of $15-\mathrm{F}_{2 \mathrm{t}}$-IsoPs with enzyme-linked immunosorbent assay (ELISA) with chromatographic methods in human plasma and urine. They noticed that commercially available ELISA kit grossly overestimated the concentration of $15-\mathrm{F}_{2 \mathrm{t}}$-IsoP especially in plasma, even after dilution.

EIA, ELISA and RIA are commercially available as kits for many eicosanoids, are easy to use, sensitive (about $23 \mathrm{pM}$ ), and allow rapid analysis of a large number of samples within a short time. 
In addition, EIA and RIA do not require expensive instrumentation such as GC-MS, GC-MS/MS, LC-MS and LC-MS/MS. EIA and RIA are therefore very often used in large experimental and clinical studies. Immunoassays are widely employed for $15-\mathrm{F}_{2 \mathrm{t}}-$ IsoP determination, in urine, plasma, CSF or EBC in correlation studies between lipid peroxidation level and oxidative stress due to numerous environmental or pathological conditions including, smoking, air pollution, diabetes, preeclampsia, hypercholesterolemia, atherosclerosis or neurodegenerative disease [96-104]. However, unspecificity due to cross-reactivity and for other poorly recognized reasons represents, besides limitation to particular eicosanoids, the most important disadvantage of EIA and RIA compared to mass spectrometry-based approaches for eicosanoids [129] and other biomarkers of oxidative stress such as 3-nitrotyrosine [130].

\section{Future perspectives}

GC-MS(/MS) coupled first with EI and later with NICI was essential in discovering the PG-like compounds IsoPs or NeuroPs, cyclic metabolites of n-6 and n-3 PUFAs, respectively, in biological samples, and in studying their in vitro and in vivo formation, and evaluating the role of this important class of oxidized lipids in human physiology and pathology. Measurement of these compounds in biological samples by GC-MS and GC-MS/MS has allowed defining them as specific biomarkers of lipid peroxidation, to evaluate the extent of oxidative stress related to human diseases, and to define reference values and intervals for IsoPs and NeuroPs. Combined measurement of multiple metabolites increased information in biological systems related to vital organs and oxidative stress status $[131,132]$.

In general, LC-MS/MS is regarded as a more versatile analytical technique compared to GC-MS and GC-MS/MS methodologies. LC-MS/MS will surely be very valuable in future platform technologies for the analysis of oxidative stress biomarkers including IsoPs and NeuroPs. At present, LC-MS/MS seems to take the liberty of dispensing with time-consuming steps in sample preparation including eicosanoids extraction and their subsequent derivatization. However, despite recent great advances in LC-MS/MS instrumentation including ionization and ions transfer, LC-MS/MS has not yet achieved the sensitivity of GC-MS/MS when performed in the NICI mode. To date, many different GC-MS/MS or LC-MS/MS methods are available for PGs, IsoPs and NeuroPs. However, reliable comparison of these techniques with respect to accuracy, one of the most important analytical features in quantitative analysis, has not been performed thus far, but it is warranted. For the time being, the use of LC-MS/MS and GC-MS/MS methods in the analysis of eicosanoids and their oxidatively modified metabolites will continue in parallel. As the older and more experienced approach in eicosanoids analysis, GC-MS/MS may serve as a landmark in the further improvement of the LC-MS/MS methodology.

Immunoassays are less specific than LC-MS/MS and GC-MS/MS assays with regard to current native and oxidatively modified eicosanoids analysis. It is expected that immunoassays will constitute important analytical tools in the analysis of a large number of samples. It is also expected that immunoassays will further improve by generating antibodies of high specificity and affinity for a wider spectrum of eicosanoids. In this context, the chemical synthesis of oxidized lipids in the highest available chemical and optical purity is indispensable.

Oxidative stress is a key feature in a number of human diseases, because ROS are likely to be involved in all disease stages. It is therefore important to identify and evaluate reliably biomarkers that allow us to monitor and quantify oxidative stress status in healthy and diseased individuals. $\mathrm{F}_{2}$-IsoPs are widely used in biological samples and have been recognized as "gold markers" of non-enzymatic lipid peroxidation. In the future, other nonenzymatic metabolites such as isofuranoids will be important to complete the panel of oxidative stress biomarkers. In this article, we reviewed and discussed the analysis of oxidized lipid metabolites in different biological samples by GC-MS(MS) and LC-MS/MS. To our understanding, LC-MS/MS methods are currently more specific and versatile methods with respect to the quantification of all series of these metabolites, whereas especially GC-MS/MS methods are more sensitive.

\section{References}

[1] B. Halliwell, J.M.C. Gutteridge (Eds.), Free Radicals in Biology and Medicine, fourth ed., Oxford University Press, Oxford, United Kingdom, 2007.

[2] U. Jahn, J.M. Galano, T. Durand, Angew. Chem. Int. Ed. 47 (2008) 5894-5955.

[3] G.L. Milne, H. Yin, K.D. Hardy, S.S. Davies, L.J. Roberts, Chem. Rev. 111 (2011) 5973-5996.

[4] G.L. Milne, B. Gao, E.S. Terry, W.E. Zackert, S.C. Sanchez, Free Radic. Biol. Med. 59 (2013) 36-44

[5] J.D. Morrow, T.M. Harris, L. Jackson Roberts II, Anal. Biochem. 184 (1990) 1-10.

[6] J.D. Morrow, L.J. Roberts, Methods Enzymol. 233 (1994) 163-174.

[7] J. Morrow, J. Awad, H. Boss, I. Blair, L. Roberts, Proc. Natl. Acad. Sci. U.S.A. 89 (1992) 10721-10725

[8] M.B. Kadiiska, B.C. Gladen, D.D. Baird, D. Germolec, L.B. Graham, C.E. Parker, A Nyska, J.T. Wachsman, B.N. Ames, S. Basu, N. Brot, G.A. FitzGerald, R.A. Floyd, M. George, J.W. Heinecke, G.E. Hatch, K. Hensley, J.A. Lawson, L.J. Marnett, J.D. Morrow, D.M. Murray, J. Plastaras, L.J. Roberts II, J. Rokach, M.K. Shigenaga, R.S. Sohal, J. Sun, R.R. Tice, D.H. Van Thiel, D. Wellner, P.B. Walter, K.B. Tomer R.P. Mason, J.C. Barrett, Free Radic. Biol. Med. 38 (2005) 698-710.

[9] EFSA Panel on Dietetic Products, Nutrition and Allergies (NDA), EFSA J. 9 (2011) 2474-2486

[10] D.F. Taber, L.J. Roberts, Prostaglandins Other Lipid Mediat. 78 (2005) 14-18.

[11] J. Rokach, S.P. Khanapure, S.W. Hwang, M. Adiyaman, J.A. Lawson, G.A. FitzGerald, Prostaglandins 54 (1997) 853-873.

[12] D.M. Stafforini, J.R. Sheller, T.S. Blackwell, A. Sapirstein, F.E. Yull, T.M. McIntyre, J.V. Bonventre, S.M. Prescott, L.J. Roberts, J. Biol. Chem. 281 (2006) 4616-4623.

[13] N. Kono, T. Inoue, Y. Yoshida, H. Sato, T. Matsusue, H. Itabe, E. Niki, J. Aoki, H Arai, J. Biol. Chem. 283 (2008) 1628-1636

[14] J.M. Proudfoot, A.E. Barden, W.M. Loke, K.D. Croft, I.B. Puddey, T.A. Mori, J Lipid Res. 50 (2009) 716-722.

[15] Z. Yan, E. Mas, T.A. Mori, K.D. Croft, A.E. Barden, Anal. Biochem. 403 (2010) $126-128$.

[16] H. Li, J.A. Lawson, M. Reilly, M. Adiyaman, S.-W. Hwang, J. Rokach, G.A. FitzGerald, Proc. Natl. Acad. Sci. U.S.A. 96 (1999) 13381-13386.

[17] J.D. Morrow, T.A. Minton, C.R. Mukundan, M.D. Campbell, W.E. Zackert, V.C. Daniel, K.F. Badr, I.A. Blair, L.J. Roberts, J. Biol. Chem. 269 (1994) 4317-4326.

[18] Y. Chen, J.D. Morrow, L.J. Roberts, J. Biol. Chem. 274 (1999) 10863-10868.

[19] R.J. Waugh, R.C. Murphy, J. Am. Soc. Mass Spectrom. 7 (1996) 490-499.

[20] R.J. Waugh, J.D. Morrow, L.J. Roberts, R.C. Murphy, Free Radic. Biol. Med. 23 (1997) 943-954

[21] H. Yin, C.M. Havrilla, J.D. Morrow, N.A. Porter, J. Am. Chem. Soc. 124 (2002) $7745-7754$.

[22] H. Yin, J.D. Morrow, N.A. Porter, J. Biol. Chem. 279 (2004) 3766-3776.

[23] M. VanRollins, R.L. Woltjer, H. Yin, J.D. Morrow, T.J. Montine, J. Lipid Res. 49 (2008) 995-1005.

[24] C. De Felice, C. Signorini, T. Durand, C. Oger, A. Guy, V. Bultel-Ponce, J.M Galano, L. Ciccoli, S. Leoncini, M. D’Esposito, S. Filosa, A. Pecorelli, G. Valacchi, J. Hayek, J. Lipid Res. 52 (2011) 2287-2297.

[25] C. De Felice, C. Signorini, T. Durand, L. Ciccoli, S. Leoncini, M. D’Esposito, S. Filosa, C. Oger, A. Guy, V. Bultel-Ponce, J.M. Galano, A. Pecorelli, L. De Felice, G. Valacchi, J. Hayek, Genes Nutr. 7 (2012) 447-458.

[26] J. Nourooz-Zadeh, B. Halliwell, E.E. Anggård, Biochem. Biophys. Res. Commun. 236 (1997) 467-472.

[27] W.L. Song, G. Paschos, S. Fries, M.P. Reilly, Y. Yu, J. Rokach, C.T. Chang, P. Patel J.A. Lawson, G.A. Fitzgerald, J. Biol. Chem. 284 (2009) 23636-23643.

[28] L. Gao, H. Yin, G.L. Milne, N.A. Porter, J.D. Morrow, J. Biol. Chem. 281 (2006) 14092-14099.

[29] J.A. Lawson, S. Kim, W.S. Powell, G.A. FitzGerald, J. Rokach, J. Lipid Res. 47 (2006) 2515-2524.

[30] C.T. Chang, P. Patel, N. Kang, J.A. Lawson, W.L. Song, W.S. Powell, G.A. FitzGerald, J. Rokach, Bioorg. Med. Chem. Lett. 18 (2008) 5523-5527.

[31] H. Yin, E.S. Musiek, L. Gao, N.A. Porter, J.D. Morrow, J. Biol. Chem. 280 (2005) 26600-26611.

[32] R. Imbusch, M.J. Mueller, Free Radic. Biol. Med. 28 (2000) 720-726.

[33] A.E. Barden, K.D. Croft, T. Durand, A. Guy, M.J. Mueller, T.A. Mori, J. Nutr. 139 (2009) 1890-1895.

[34] K. Karg, V.M. Dirsch, A.M. Vollmar, J.L. Cracowski, F. Laporte, M.J. Mueller, Free Radic. Res. 41 (2007) 25-37.

[35] A.E. Barden, E. Mas, K.D. Croft, M. Phillips, T.A. Mori, Anal. Biochem. 449 (2014) $129-131$ 
[36] M.L. Langhorst, M.J. Hastings, W.H. Yokoyama, S.C. Hung, N. Cellar, K. Kuppannan, S.A. Young, J. Agric. Food Chem. 58 (2010) 6614-6620.

[37] S.A. Brose, A.G. Baker, M.Y. Golovko, Lipids 48 (2013) 411-419.

[38] C.Y. Lee, A.M. Jenner, B. Halliwell, Biochem. Biophys. Res. Commun. 320 (2004) 696-702.

[39] Y. Yoshida, M. Hayakawa, Y. Habuchi, N. Itoh, E. Niki, Lipids 42 (2007) 463-472.

[40] C.F. Labuschagne, E.C.A. Stigter, M.M.W.B. Hendriks, R. Berger, J. Rokach, H.C. Korswagen, A.B. Brenkman, Aging Cell 12 (2013) 214-223.

[41] X. Liu, P.D. Whitefield, Y. Ma, Talanta 81 (2010) 1599-1606.

[42] K.S. Leung, X. Chen, W. Zhong, A.C.H. Yu, C.Y.J. Lee, Chem. Phys. Lipids 180 (2014) 53-60.

[43] B. Halliwell, C.Y. Lee, Antioxid. Redox Signal. 13 (2010) 145-156.

[44] D. Milatovic, T.J. Montine, M. Aschner, Methods Mol. Biol. 758 (2011) 195-204.

[45] C.F. Labuschagne, A.B. Brenkman, Ageing Res. Rev. 12 (2013) 918-930.

[46] J.K. Prasain, A. Arabshahi, P.R. Taub, S. Sweeney, R. Moore, J.D. Sharer, S. Barnes, J. Chromatogr. B 913/914 (2013) 161-168.

[47] W. Liu, J.D. Morrow, H. Yin, Free Radic. Biol. Med. 47 (2009) 1101-1107.

[48] M. Haschke, Y.L. Zhang, C. Kahle, J. Klawitter, M. Korecka, L.M. Shaw, U. Christians, Clin. Chem. 53 (2007) 489-497.

[49] D. Tsikas, E. Schwedhelm, M.-T. Suchy, J. Niemann, F.-M. Gutzki, V.J. Erpenbeck, J.M. Hohlfeld, A. Surdacki, J.C. Frölich, J. Chromatogr. B 794 (2003) 237-255.

[50] O. Berdeaux, O. Scruel, J.L. Cracowski, T. Durand, Curr. Pharm. Anal. 2 (2006) 69-78.

[51] Y.-P. Hsieh, C.-L. Lin, A.-L. Shiue, H. Yin, J.D. Morrow, J.-C. Hsu, T.-C. Hsieh, H.-J. Wei, H.-C. Yen, Free Radic. Biol. Med. 47 (2009) 814-824

[52] J.-U. Grundmann, I. Wiswedel, D. Hirsch, H.P.M. Gollnick, Skin Pharmacol. Physiol. 17 (2004) 37-41.

[53] C. Kupfahl, D. Tsikas, J. Niemann, G. Geginat, H. Hof, Mol. Immunol. 49 (2012) 621-627.

[54] Y. Yao, V. Zhukareva, S. Sung, C.M. Clark, J. Rokach, V.M.-Y. Lee, J.Q. Trojanowski, D. Praticò, Neurology 61 (2003) 475-478.

[55] C.-Y.J. Lee, R.C.S. Seet, S.H. Huang, L.H. Long, B. Halliwell, Antioxid. Redox Signal. 11 (2009) 407-420.

[56] L.J. Roberts, K.P. Moore, W.E. Zackert, J.A. Oates, J.D. Morrow, J. Biol. Chem. 271 (1996) 20617-20620.

[57] C. Chiabrando, A. Valagussa, C. Rivalta, T. Durand, A. Guy, E. Zuccato, P. Villa, J.C. Rossi, R. Fanelli, J. Biol. Chem. 274 (1999) 1313-1319.

[58] C.R. Morales, E.S. Terry, W.E. Zackert, T.J. Montine, J.D. Morrow, Clin. Chim. Acta 314 (2001) 93-99.

[59] J. Nourooz-Zadeh, M.B. Cooper, D. Ziegler, D.J. Betteridge, Biophys. Res. Commun. 330 (2005) 731-736.

[60] T. Dorjgochoo, Y.T. Gao, W.H. Chow, X.O. Shu, G. Yang, Q. Cai, N. Rothman, H. Cai, H. Li, X. Deng, A. Franke, L.J. Roberts, G. Milne, W. Zheng, Q. Dai, Am. J. Clin. Nutr. 96 (2012) 405-414.

[61] C. Oger, V. Bultel-Poncé, A. Guy, T. Durand, J. Galano, Eur. J. Org. Chem. 2012 (2012) 2621-2634.

[62] C. Signorini, C.D. Felice, S. Leoncini, A. Giardini, M. D’Esposito, S. Filosa, F.D. Ragione, M. Rossi, A. Pecorelli, G. Valacchi, L. Ciccoli, J. Hayek, Clin. Chim. Acta 412 (2011) 1399-1406.

[63] L.J. Roberts, T.J. Montine, W.R. Markesbery, A.R. Tapper, P. Hardy, S. Chemtob, W.D. Dettbarn, J.D. Morrow, J. Biol. Chem. 273 (1998) 13605-13612.

[64] J. Nourooz-Zadeh, E.H. Liu, B. Yhlen, E.E. Anggard, B. Halliwell, J. Neurochem. 72 (1999) 734-740.

[65] T.B. Corcoran, E. Mas, A.E. Barden, T. Durand, J.M. Galano, L.J. Roberts, M. Phillips, K.M. Ho, T.A. Mori, Antioxid. Redox Signal. 15 (2011) 2663-2667.

[66] H.-C. Yen, H.-J. Wei, T.-W. Chen, BioMed Res. Int. 2013 (2013)

[67] A.E. Barden, T.B. Corcoran, E. Mas, T. Durand, J.M. Galano, L.J. Roberts, M. Paech, N.A. Muchatuta, M. Phillips, T.A. Mori, Antioxid. Redox Signal. 16 (2012) $165-169$.

[68] E.S. Musiek, J.K. Cha, H. Yin, W.E. Zackert, E.S. Terry, N.A. Porter, T.J. Montine, J.D. Morrow, J. Chromatogr. B 799 (2004) 95-102.

[69] C. Signorini, L. Ciccoli, S. Leoncini, S. Carloni, S. Perrone, M. Comporti, W. Balduini, G. Buonocore, J. Pineal Res. 46 (2009) 148-154.

[70] E.E. Reich, W.E. Zackert, C.J. Brame, Y. Chen, L.J. Roberts, D.L. Hachey, T.J. Montine, J.D. Morrow, Biochemistry 39 (2000) 2376-2383.

[71] E.E. Reich, W.R. Markesbery, L.J. Roberts Ii, L.L. Swift, J.D. Morrow, T.J. Montine, Am. J. Pathol. 158 (2001) 293-297.

[72] L.J. Roberts II, J.P. Fessel, Chem. Phys. Lipids 128 (2004) 173-186.

[73] S.S. Fam, L.J. Murphey, E.S. Terry, W.E. Zackert, Y. Chen, L. Gao, S. Pandalai, G.L. Milne, L.J. Roberts, N.A. Porter, T.J. Montine, J.D. Morrow, J. Biol. Chem. 277 (2002) 36076-36084.

[74] S. Parchmann, M.J. Mueller, J. Biol. Chem. 273 (1998) 32650-32655.

[75] I. Thoma, M. Krischke, C. Loeffler, M.J. Mueller, Chem. Phys. Lipids 128 (2004) $135-148$.

[76] T. Durand, V. Bultel-Poncé, A. Guy, S. Berger, M. Mueller, J.-M. Galano, Lipids 44 (2009) 875-888.

[77] K.A. Massey, A. Nicolaou, Free Radic. Biol. Med. 59 (2013) 45-55.

[78] D.D. Shinde, K.-B. Kim, K.-S. Oh, N. Abdalla, K.-H. Liu, S.K. Bae, J.-H. Shon, H.-S. Kim, D.-H. Kim, J.G. Shin, J. Chromatogr. B 911 (2012) 113-121.

[79] K. Sterz, G. Scherer, J. Ecker, J. Lipid Res. 53 (2012) 1026-1036.

[80] J. Klawitter, M. Haschke, T. Shokati, U. Christians, Rapid Commun. Mass Spectrom. 25 (2011) 463-468.

[81] J.D. Morrow, L.J. Roberts, V.C. Daniel, J.A. Awad, O. Mirochnitchenko, L.L. Swift, R.F. Burk, Arch. Biochem. Biophys. 353 (1998) 160-171.
[82] S.E. Farias, M. Basselin, L. Chang, K.A. Heidenreich, S.I. Rapoport, R.C. Murphy, J. Lipid Res. 49 (2008) 1990-2000.

[83] S.A. Brose, B.T. Thuen, M.Y. Golovko, J. Lipid Res. 52 (2011) 850-859.

[84] S.M. Lamos, M.R. Shortreed, B.L. Frey, P.J. Belshaw, L.M. Smith, Anal. Chem. 79 (2007) 5143-5149.

[85] W.-C. Yang, J. Adamec, F.E. Regnier, Anal. Chem. 79 (2007) 5150-5157.

[86] C. Pettinella, S.H. Lee, F. Cipollone, I.A. Blair, J. Chromatogr. B 850 (2007) $168-176$.

[87] J.G. Bollinger, G. Rohan, M. Sadilek, M.H. Gelb, J. Lipid Res. 54 (2013) 3523-3530.

[88] H. Yin, N.A. Porter, J.D. Morrow, J. Chromatogr. B 827 (2005) $157-164$.

[89] D. Pratico, O.P. Barry, J.A. Lawson, M. Adiyaman, S.W. Hwang, S.P. Khanapure L. Iuliano, J. Rokach, G.A. FitzGerald, Proc. Natl. Acad. Sci. U.S.A. 95 (1998) 3449-3454

[90] F. Mangialasche, M.C. Polidori, R. Monastero, S. Ercolani, C. Camarda, R. Cecchetti, P. Mecocci, Ageing Res. Rev. 8 (2009) 285-305.

[91] M. Korecka, C.M. Clark, V.M.-Y. Lee, J.Q. Trojanowski, L.M. Shaw, J. Chromatogr. B 878 (2010) 2209-2216.

[92] J.D. Brooks, G.L. Milne, H. Yin, S.C. Sanchez, N.A. Porter, J.D. Morrow, J. Biol. Chem. 283 (2008) 12043-12055.

[93] M.L.S. Chung, K.Y.E. Lee, C.-Y.J. Lee, Food Chem. Toxicol. 53 (2013) 205-213.

[94] S. Basu, Prostaglandins Leukot. Essent. Fatty Acids 58 (1998) 319-325.

[95] Z. Wang, G. Ciabattoni, C. Creminon, J. Lawson, G.A. Fitzgerald, C. Patrono, J. Maclouf, J. Pharmacol. Exp. Ther. 275 (1995) 94-100.

[96] P. Rossner Jr., A. Milcova, H. Libalova, Z. Novakova, J. Topinka, I. Balascak, R.J. Sram, Mutat. Res.: Fundam. Mol. Mech. Mutagen. 669 (2009) 20-26.

[97] C.-N. Wang, J.Y.-S. Chen, S. Sabu, Y.-L. Chang, S.-D. Chang, C.-C. Kao, H.-H. Peng, H.-Y. Chueh, A.-S. Chao, P.-J. Cheng, Y.-S. Lee, L.-M. Chi, T.-H. Wang, Free Radic Biol. Med. 50 (2011) 1124-1130.

[98] G. Davi, G. Ciabattoni, A. Consoli, A. Mezzetti, A. Falco, S. Santarone, E. Pennese, E. Vitacolonna, T. Bucciarelli, F. Costantini, F. Capani, C. Patrono, Circulation 99 (1999) 224-229.

[99] V. Lucidi, G. Ciabattoni, S. Bella, P.J. Barnes, P. Montuschi, Free Radic. Biol. Med. 45 (2008) 913-919.

[100] L. Loffredo, F. Martino, R. Carnevale, P. Pignatelli, E. Catasca, L. Perri, C.M Calabrese, M.M. Palumbo, F. Baratta, M. Del Ben, F. Angelico, F. Violi, J. Pediatr. 161 (2012) 1004-1009.

[101] T.J. Montine, E.R. Peskind, J.F. Quinn, A.M. Wilson, K.S. Montine, D. Galasko, Neuromolecular Med. 13 (2011) 37-43.

[102] J. Proudfoot, A. Barden, T.A. Mori, V. Burke, K.D. Croft, L.J. Beilin, I.B. Puddey, Anal. Biochem. 272 (1999) 209-215.

[103] A.K. Saenger, T.J. Laha, M.J. Edenfield, S.M.H. Sadrzadeh, Clin. Chem. 40 (2007) $1297-1304$

[104] J.H. Dahl, R.B. van Breemen, Anal. Biochem. 404 (2010) 211-216.

[105] D. Tsikas, J. Chromatogr. B 717 (1998) 201-245.

[106] J.D. Morrow, L.J. Roberts, in: L. Packer (Ed.), Methods in Enzymol., Academic Press, 1999, pp. 3-12.

[107] E. Mas, F. Michel, A. Guy, V. Bultel, Y. Falquet, P. Chardon, J.C. Rossi, J.P. Cristol, T. Durand, J. Chromatogr. B 872 (2008) 133-140.

[108] E. Mas, A. Barden, T. Durand, J.M. Galano, K.D. Croft, T.A. Mori, Free Radic. Res. 44 (2010) 191-198

[109] E. Mas, R.J. Woodman, V. Burke, I.B. Puddey, L.J. Beilin, T. Durand, T.A. Mori, Free Radic. Res. 44 (2010) 983-990.

[110] G.L. Milne, S.C. Sanchez, E.S. Musiek, J.D. Morrow, Nat. Protocols 2 (2007) 221-226.

[111] C. Signorini, M. Comporti, G. Giorgi, J. Mass Spectrom. 38 (2003) 1067-1074

[112] M.J. Mueller, L. Mene-Saffrane, C. Grun, K. Karg, E.E. Farmer, Plant J. 45 (2006) 472-489.

[113] R.C. Seet, C.Y. Lee, W.M. Loke, S.H. Huang, H. Huang, W.F. Looi, E.S Chew, A.M. Quek, E.C. Lim, B. Halliwell, Free Radic. Biol. Med. 50 (2011) 1787-1793.

[114] C. De Felice, L. Ciccoli, S. Leoncini, C. Signorini, M. Rossi, L. Vannuccini, G. Guazzi, G. Latini, M. Comporti, G. Valacchi, J. Hayek, Free Radic. Biol. Med. 47 (2009) 440-448.

[115] A. Barden, E. Mas, P. Henry, T. Durand, J.M. Galano, L.J. Roberts, K.D. Croft, T.A Mori, Free Radic. Res. 45 (2011) 469-476.

[116] L.G. Wood, M.L. Garg, J.L. Simpson, T.A. Mori, K.D. Croft, P.A.B. Wark, P.G. Gibson, Am. J. Respir. Crit. Care Med. 171 (2005) 426-430.

[117] W.F. Ward, W. Qi, H.V. Remmen, W.E. Zackert, L.J. Roberts, A. Richardson, J. Gerontol. A: Biol. Sci. Med. Sci. 60 (2005) 847-851.

[118] W.-Y. Ong, A.M. Jenner, N. Pan, C.-N. Ong, B. Halliwell, Free Radic. Res. 43 (2009) 241-249.

[119] L. Gao, W.E. Zackert, J.J. Hasford, M.E. Danekis, G.L. Milne, C. Remmert, J. Reese, H. Yin, H.H. Tai, S.K. Dey, N.A. Porter, J.D. Morrow, J. Biol. Chem. 278 (2003) 28479-28489.

[120] R. Solberg, M. Longini, F. Proietti, P. Vezzosi, O.D. Saugstad, G. Buonocore, Free Radic. Biol. Med. 53 (2012) 1061-1067.

[121] J.M. Al-Hassan, M. Ali, M. Thomson, C.R. Pace-Asciak, Prostaglandins Leukot. Essent. Fatty Acids 59 (1998) 325-328.

[122] N.E. Bastani, T.E. Gundersen, R. Blomhoff, Rapid Commun. Mass Spectrom. 23 (2009) 2885-2890. 
[123] J. Larose, P. Julien, J.-F. Bilodeau, J. Lipid Res. 54 (2013) 1505-1511.

[124] A.W. Taylor, R.S. Bruno, M.G. Traber, Lipids 43 (2008) 925-936.

[125] K. Syslová, P. Kačer, M. Kuzma, P. Klusáčková, Z. Fenclová, J. Lebedová, D. Pelclová, J. Chromatogr. B 867 (2008) 8-14.

[126] M. Janicka, A. Kot-Wasik, J. Paradziej-Łukowicz, G. Sularz-Peszyńska, A. Bartoszek, J. Namieśnik, Int. J. Mol. Sci. 14 (2013) 6157-6169.

[127] T. Sicilia, A. Mally, U. Schauer, A. Pähler, W. Völkel, J. Chromatogr. B 861 (2008) $48-55$.
[128] C.F. Labuschagne, N.J.F. van den Broek, P. Postma, R. Berger, A.B. Brenkman, PLoS ONE 8 (2013) e80935.

[129] D. Tsikas, M.T. Suchy, Hypertension 60 (2012) e14.

[130] D. Tsikas, Clin. Chem. Lab. Med. 48 (2010) 141-143.

[131] I. Dalle-Donne, R. Rossi, R. Colombo, D. Giustarini, A. Milzani, Clin. Chem. 52 (2006) 601-623.

[132] D. Giustarini, I. Dalle-Donne, D. Tsikas, R. Rossi, Crit. Rev. Clin. Lab. Sci. 46 (2009) 241-281. 